\title{
Computational Acoustics in Multi-field Problems
}

\author{
Manfred Kaltenbacher \\ Chair of Applied Mechatronics, Alps-Adriatic University of Klagenfurt, \\ Klagenfurt, 9020, Austria manfred.kaltenbacher@uni-klu.ac.at \\ http://am.uni-klu.ac.at \\ Received (Day Month Year) \\ Revised (Day Month Year)
}

\begin{abstract}
We present physical/mathematical models base on partial differential equations (PDEs) and efficient numerical simulation schemes based on the Finite Element (FE) method for multi-field problems, where the acoustic field is the field of main interest. Acoustics, the theory of sound, is an emerging scientific field including disciplines from physics over engineering to medical science. We concentrate on the following three topics: vibro-acoustics, aero-acoustics and high intensity focused ultrasound. For each topic, we discuss the physical/mathematical modeling, efficient numerical schemes and provide practical applications.
\end{abstract}

Keywords: multi-field problems; vibro-acoustics; aero-acoustics; high intensity focused ultrasound; human phonation; finite element method

\section{Introduction}

The physical/mathematical modeling and numerical simulation of complex systems in engineering and medical science is an emerging topic and a topic of intensive active research. Often these systems, e.g. sensors and/or actuators, are immersed in an acoustic fluid and the computation of the acoustic quantities such as sound pressure level (SPL), radiated sound intensity and power are of main interest. Since all the different coupling mechanisms of the involved physical fields have to be considered for the development of these systems, the design process is a very complex task. Therefore, an increasing need for reliable and usable computer modeling tools capable of precisely simulating the multi-field interactions arises. Such appropriate computer-aided engineering (CAE) tools offer many possibilities to the design engineer. Arbitrary modification of transducer geometry and selective variation of material parameters are easily performed and the influence on the transducer behavior can be studied immediately. In addition, simulations provide access to physical quantities that cannot be measured and thus strongly support the insight into physical phenomena. Therefore, CAE-based design tremendously reduces the number of necessary prototypes within the design process. However, we have to be aware of the fact, that the mathematical model is always an abstraction of reality and especially the material models and their parameters will limit the accuracy between model and reality.

Acoustics has developed into an interdisciplinary field encompassing the disciplines of physics, engineering, speech, audiology, music, architecture, psychology, neuro-science, and 
others (see, e.g., [1]). Therewith, the arising multi-field problems range from classical airborne sound over underwater acoustics (e.g., ocean acoustics) to ultrasound used in medical application. We concentrate on three topics: vibro-acoustics (Sec. 2), high intensity focused ultrasound (Sec. 3) and aero-acoustics (Sec. 4). For all three topics we will discuss the phys$\mathrm{ical} /$ mathematical models as well as efficient numerical schemes for their solution applying the Finite Element (FE) method. To demonstrate the applicability of the developed numerical schemes, we will discuss three applications: active damping by piezoelectric patches, high intensity focused ultrasound (HIFU) for treatment of cancers as well as use in lithotripsy and the human phonation process. All examples have in common, that they are multi-field problems, where the acoustic quantities are of main interest. The formulations described within this work have been successfully implemented in our research software CFS $++(\mathrm{Cou}-$ pled Field Simulation) [2].

\section{Vibro-Acoustics}

In many technical applications, sensors as well as actuators are immersed in an acoustic fluid. Therefore, mechanical vibrations will generate acoustic waves, which itself will act as a surface pressure load on the vibrating structure. In general, we distinguish between the

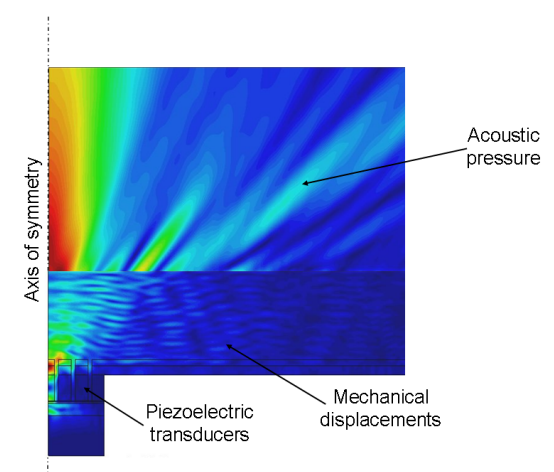

(a) Acoustic sound field of a piezoelectric ultrasound array antenna.

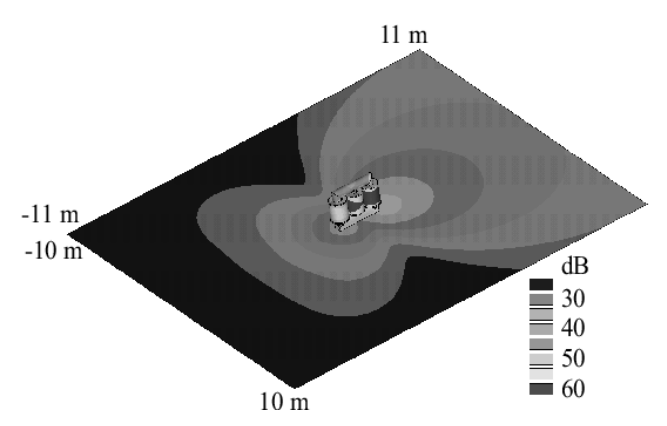

(b) Acoustic sound field of an electric power transformer due to the Lorentz forces acting on its winding.

Fig. 1. Two typical setups, where the computation of the acoustic field within the multi-field problem is the main point of interest.

following two situations concerning mechanical-acoustic systems:

- Strong Coupling:

In this case, the mechanical and acoustic field equations including their couplings have to be solved simultaneously. A typical example is a piezoelectric ultrasound array immersed in water (see Fig. 1(a) and [3]). 
- Weak Coupling:

If the acoustic pressure forces on the solid are negligible, a sequential computation can be performed. For example, the acoustic sound field of an electric transformer as displayed in Fig. 1(b) can be obtained in this way. In a first simulation the mechanical surface vibrations are calculated which are used as the input for a subsequent acoustic field computation [4].

At a solid-fluid interface $\Gamma_{\mathrm{sa}}$, the continuity requires that the normal component of the mechanical surface velocity must coincide with the normal component of the acoustic particle velocity of the fluid. Thus, the following relation between the velocity $\mathbf{v}_{\mathbf{s}}$ of the solid expressed by the mechanical displacement $\mathbf{u}$ and the acoustic particle velocity $\mathbf{v}^{\prime}$ expressed by the acoustic scalar potential $\psi$ arises

$$
\begin{gathered}
\mathbf{v}_{\mathrm{s}}=\frac{\partial \mathbf{u}}{\partial t} \quad \mathbf{v}^{\prime}=-\boldsymbol{\nabla} \psi \\
\mathbf{n}_{\mathrm{s}} \cdot\left(\mathbf{v}_{\mathrm{s}}-\mathbf{v}^{\prime}\right)=0 \\
\mathbf{n}_{\mathrm{s}} \cdot \frac{\partial \mathbf{u}}{\partial t}=-\mathbf{n}_{\mathrm{s}} \cdot \boldsymbol{\nabla} \psi=-\frac{\partial \psi}{\partial \mathbf{n}_{\mathrm{s}}} .
\end{gathered}
$$

In (1) $\mathbf{n}_{\mathrm{s}}$ denotes the normal vector on $\Gamma_{\mathrm{sa}}$ pointing from the solid to the fluid domain. In addition, one has to consider the fact that the ambient fluid causes a mechanical stress $\boldsymbol{\sigma}_{\mathrm{n}}$ on the surface

$$
\boldsymbol{\sigma}_{\mathrm{n}}=-\mathbf{n}_{\mathrm{s}} p^{\prime}=-\mathbf{n}_{\mathrm{s}} \rho_{0} \frac{\partial \psi}{\partial t}
$$

which acts as a surface load, resulting from the acoustic pressure $p^{\prime}$. In $(2) \rho_{0}$ denotes the mean density of the fluid.

When modeling special wave phenomena, we often arrive at a partial differential equation for the acoustic pressure. Therewith, we will also derive the coupling conditions between the mechanical displacement $\mathbf{u}$ and acoustic pressure $p^{\prime}$ at a solid-fluid interface. For the first coupling condition, the continuity of the velocities, we have to establish the relation between the acoustic particle velocity $\mathbf{v}^{\prime}$ and the acoustic pressure $p^{\prime}$. According to the linearized momentum equation for acoustics (see, e.g., [5]), we can express the normal component of $\mathbf{v}^{\prime}$ by

$$
\mathbf{n}_{\mathrm{s}} \cdot \frac{\partial \mathbf{v}^{\prime}}{\partial t}=-\frac{1}{\rho_{0}} \frac{\partial p^{\prime}}{\partial \mathbf{n}_{\mathrm{s}}}
$$

Since $\mathbf{n}_{\mathrm{s}} \cdot \mathbf{v}_{\mathrm{s}}=\mathbf{n}_{\mathrm{s}} \cdot \mathbf{v}^{\prime}$ has to hold, we get the relation for the mechanical displacement by

$$
\mathbf{n}_{\mathrm{s}} \cdot \frac{\partial^{2} \mathbf{u}}{\partial t^{2}}=-\frac{1}{\rho_{0}} \frac{\partial p^{\prime}}{\partial \mathbf{n}_{\mathrm{s}}}
$$

The second coupling condition defined in (2) is already established for an acoustic pressure formulation. 


\subsection{FE Formulation for Vibro-Acoustics}

The acoustic part of the computational domain $\Omega_{\mathrm{a}} \subset \mathbb{R}^{d}$ as depicted in Fig. 2(a) is governed by the linear acoustic wave equation in pressure formulation. The strong form reads as

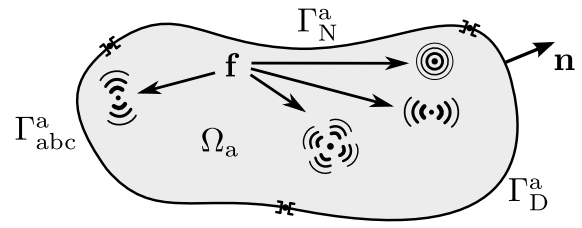

(a) Geometrical setup for acoustics.

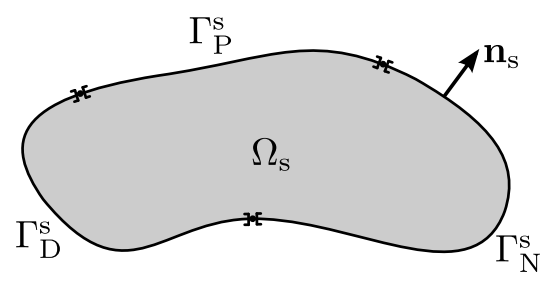

(b) Geometrical setup for mechanics.

Fig. 2. Computational domains for acoustics and mechanics.

follows: Given

$$
p_{0}^{\prime}, \dot{p}_{0}^{\prime}: \Omega_{\mathrm{a}} \rightarrow \mathbb{R},
$$

find the acoustic pressure $p^{\prime}: \overline{\Omega_{\mathrm{a}}} \times[0, T] \rightarrow \mathbb{R}$ such that

$$
\Delta p^{\prime}-\frac{1}{c^{2}} \frac{\partial^{2} p^{\prime}}{\partial t^{2}}=0 \text { in } \Omega_{\mathrm{a}} \times(0, T),
$$

with Dirichlet and Neumann boundary conditions of the form

$$
p^{\prime}=p_{\mathrm{D}}^{\prime} \quad \text { on } \quad \Gamma_{\mathrm{D}}^{\mathrm{a}} \times(0, T) ; \quad \frac{\partial p^{\prime}}{\partial \mathbf{n}}=p_{\mathrm{N}}^{\prime} \quad \text { on } \quad \Gamma_{\mathrm{N}}^{\mathrm{a}} \times(0, T),
$$

and initial conditions

$$
p^{\prime}(\mathbf{x}, 0)=p_{0}^{\prime}, \dot{p}^{\prime}(\mathbf{x}, 0)=\dot{p}_{0}^{\prime}, \mathbf{x} \in \Omega_{\mathrm{a}} .
$$

In (5) $c: \Omega_{\mathrm{a}} \rightarrow \mathbb{R}$ denotes the speed of sound which is assumed to be constant.

One of the great challenges for each volume discretization scheme is the precise modeling of unbounded domains. The crucial point for these computations is that the numerical scheme avoids any reflections at the boundaries, even for the case where the computational domain is just a fraction of a wavelength. Since the beginning of the eighties of the last century, several numerical techniques have been developed to deal with this topic: infinite elements, Dirichlet-to-Neumann operators based on truncated Fourier expansions, absorbing boundary conditions, perfectly matched layer (PML) technique, etc. For an overview on this topic, we refer to [6]. To simplify our derivation, we will apply an absorbing boundary condition of first order (cf. [7]) on $\Gamma_{\mathrm{abc}}^{\mathrm{a}}$, where a free field radiation condition shall be considered

$$
\left(\frac{\partial}{\partial t}-c \frac{\partial}{\partial \mathbf{n}}\right) p^{\prime}=0 \text { on } \Gamma_{\mathrm{abc}}^{\mathrm{a}}
$$


Multiplying (5) by an appropriate test function, integrating over the whole computational domain and perform an integration by parts will lead to the weak formulation: Find $p^{\prime} \in$ $V=\left\{\varphi \in H^{1}\left(\Omega_{\mathrm{a}}\right) \mid \varphi=p_{\mathrm{D}} \text { on } \Gamma_{\mathrm{D}}^{\mathrm{a}}\right\}^{\mathrm{a}}$ such that

$$
\int_{\Omega_{\mathrm{a}}} \frac{1}{c^{2}} w \ddot{p}^{\prime} \mathrm{d} \Omega+\int_{\Omega_{\mathrm{a}}} \nabla w \cdot \nabla p^{\prime} \mathrm{d} \Omega-\int_{\Gamma_{\mathrm{N}}^{\mathrm{a}}} w p_{\mathrm{N}}^{\prime} \mathrm{d} \Gamma-\int_{\Gamma_{\mathrm{abc}}^{\mathrm{a}}} \frac{1}{c} w \frac{\partial p^{\prime}}{\partial t} \mathrm{~d} \Gamma=0
$$

for all $w \in W=\left\{q \in H^{1}\left(\Omega_{\mathrm{a}}\right) \mid q=0\right.$ on $\left.\Gamma_{\mathrm{D}}^{\mathrm{a}}\right\}$.

Furthermore, we consider the problem of a solid elastic body in the domain $\Omega_{\mathrm{s}} \subset \mathbb{R}^{d}, d=$ 2,3 as shown in Fig. 2(b). The body has an isotropic density $\rho_{\mathrm{s}}: \Omega_{\mathrm{s}} \rightarrow \mathbb{R}$ and deforms under the given time dependent volume force $\mathbf{f}_{\mathrm{V}}$ and the surface traction $\mathbf{g}$. The surface of $\Omega_{\mathrm{S}}$ with the outward normal vector $\mathbf{n}_{\mathrm{S}}$ consists of the disjoint subsets $\Gamma_{\mathrm{D}}^{\mathrm{s}}$, where Dirichlet boundary conditions are given, $\Gamma_{\mathrm{N}}^{\mathrm{s}}$ a Neumann boundary with given surface traction, and $\Gamma_{\mathrm{P}}^{\mathrm{s}}$ a Neumann boundary with prescribed pressure load. The linear differential operator $\mathcal{B}$

$$
\mathcal{B}^{\mathrm{t}}=\left(\begin{array}{cccccc}
\frac{\partial}{\partial x} & 0 & 0 & 0 & \frac{\partial}{\partial z} & \frac{\partial}{\partial y} \\
0 & \frac{\partial}{\partial y} & 0 & \frac{\partial}{\partial z} & 0 & \frac{\partial}{\partial x} \\
0 & 0 & \frac{\partial}{\partial z} & \frac{\partial}{\partial y} & \frac{\partial}{\partial x} & 0
\end{array}\right)
$$

relates the displacement field $\mathbf{u}$ to the strains. The material law describing the relationship between strains and stresses is given by the elasticity tensor [c] with component functions $c_{i j k l}: \Omega_{\mathrm{s}} \rightarrow \mathbb{R}$. The strong formulation of the mechanical problem therefore reads as follows: Given initial conditions and mechanical volume force

$$
\mathbf{u}_{0}, \dot{\mathbf{u}}_{0}, \mathbf{f}_{\mathrm{V}}: \Omega_{\mathrm{s}} \rightarrow \mathbb{R}^{d},
$$

find mechanical displacement $\mathbf{u}: \overline{\Omega_{\mathrm{s}}} \times[0, T] \rightarrow \mathbb{R}^{d}$ such that

$$
\mathcal{B}^{\mathrm{t}}[\mathbf{c}] \mathcal{B} \mathbf{u}+\mathbf{f}_{\mathrm{V}}=\rho_{\mathrm{s}} \frac{\partial^{2} \mathbf{u}}{\partial t^{2}} \text { in } \Omega_{\mathrm{s}} \times(0, T)
$$

with boundary conditions

$$
\begin{array}{rll}
\mathbf{u}=\mathbf{u}_{\mathrm{D}} & \text { on } & \Gamma_{\mathrm{D}}^{\mathrm{s}} \times(0, T), \\
\boldsymbol{\sigma}_{\mathrm{t}}=\mathbf{g} & \text { on } & \Gamma_{\mathrm{N}}^{\mathrm{s}} \times(0, T), \\
{[\boldsymbol{\sigma}] \cdot \mathbf{n}_{\mathrm{s}}=p_{\mathrm{n}} \mathbf{n}_{\mathrm{s}}} & \text { on } & \Gamma_{\mathrm{P}}^{\mathrm{s}} \times(0, T),
\end{array}
$$

and initial conditions

$$
\mathbf{u}(\mathbf{x}, 0)=\mathbf{u}_{0}, \mathbf{x} \in \Omega_{\mathrm{s}} ; \quad \dot{\mathbf{u}}(\mathbf{x}, 0)=\dot{\mathbf{u}}_{0}, \mathbf{x} \in \Omega_{\mathrm{s}},
$$

${ }^{\mathrm{a}} \mathrm{H}^{1}$ is the Sobolev space, i.e. space of square integrable functions, whose first derivatives in a weak sense are also square integrable (see [8]) 
where $[\boldsymbol{\sigma}]$ denotes the tensor of Cauchy stresses, $p_{\mathrm{n}} \mathbf{n}_{\mathrm{S}}$ is an external pressure load acting in normal direction on $\Gamma_{\mathrm{P}}^{\mathrm{s}}$, whereas $\mathbf{g}$ is a surface traction acting in arbitrary direction on $\Gamma_{\mathrm{N}}^{\mathrm{s}}$.

Multiplying (8) by an appropriate test function $\mathbf{w}$, integrating over the whole computational domain and perform an integration by parts results in the weak formulation:

Find $\mathbf{u} \in \mathbf{V}=\left\{\mathbf{v} \in\left(H^{1}\right)^{d}\left(\Omega_{\mathrm{s}}\right) \mid \mathbf{v}=\mathbf{u}_{\mathrm{D}}\right.$ on $\left.\Gamma_{\mathrm{D}}^{\mathrm{s}}\right\}$, such that

$$
\int_{\Omega_{\mathrm{s}}} \rho_{\mathrm{s}} \mathbf{w} \cdot \ddot{\mathbf{u}} \mathrm{d} \Omega+\int_{\Omega_{\mathrm{s}}}(\mathcal{B} \mathbf{w})^{\mathrm{t}}[\mathbf{c}] \mathcal{B} \mathbf{u} \mathrm{d} \Omega=\int_{\Omega_{\mathrm{s}}} \mathbf{w} \cdot \mathbf{f}_{\mathrm{V}} \mathrm{d} \Omega+\int_{\Gamma_{\mathrm{P}}^{\mathrm{s}}} p_{\mathrm{n}} \mathbf{w} \cdot \mathbf{n}_{\mathrm{s}} \mathrm{d} \Gamma+\int_{\Gamma_{\mathrm{N}}^{\mathrm{s}}} \mathbf{w} \cdot \mathbf{g}_{\mathrm{n}} \mathrm{d} \Gamma
$$

for all $\mathbf{w} \in \mathbf{W}=\left\{\mathbf{q} \in\left(H^{1}\right)^{d}\left(\Omega_{\mathrm{s}}\right) \mid \mathbf{q}=\mathbf{0}\right.$ on $\left.\Gamma_{\mathrm{D}}^{\mathrm{s}}\right\}$. The mechanical and the acoustic fields are coupled along a common interface $\Gamma_{\text {sa }}$ (see Fig. 3(a)). We assume that the common

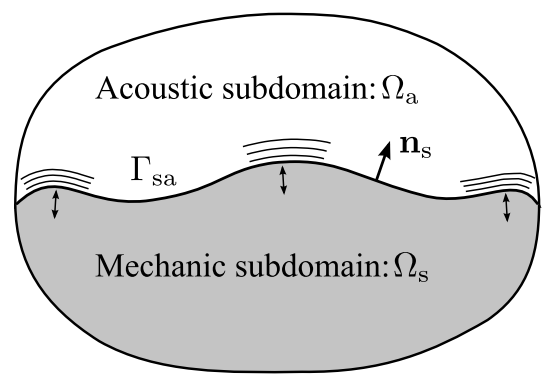

(a) Solid-fluid interface.

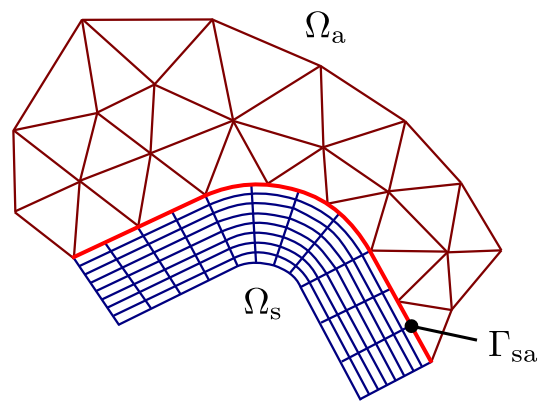

(b) Nonmatching grids along mechanical-acoustic interface $\Gamma_{\text {sa }}$.

Fig. 3. Solid fluid interface: general setup and FE mesh with a nonconforming interface.

interface $\Gamma_{\mathrm{sa}}$ corresponds to $\Gamma_{\mathrm{N}}^{\mathrm{s}}$ in (9) and to $\Gamma_{\mathrm{N}}^{\mathrm{a}}$ in $(7)$, so that we have $\Gamma_{\mathrm{sa}}=\Gamma_{\mathrm{N}}^{\mathrm{s}}=$ $\Gamma_{N}^{a}$. We may therefore substitute the surface integral term over $\Gamma_{N}^{\mathrm{s}}$ in (9) by the pressure interface condition (2) and the surface integral term over $\Gamma_{\mathrm{N}}^{\mathrm{a}}$ in (7) by the velocity interface condition (4). Furthermore, for simplicity we set $\mathbf{f}_{\mathrm{V}}$ and $\mathbf{g}$ to zero, and arrive at the fully coupled system where additionally an external pressure load $p_{\mathrm{n}}$ is applied to the mechanical subsystem

$$
\begin{array}{r}
\int_{\Omega_{\mathrm{s}}} \rho_{\mathrm{s}} \mathbf{w} \cdot \ddot{\mathbf{u}} \mathrm{d} \Omega+\int_{\Omega_{\mathrm{s}}}(\mathcal{B} \mathbf{w})^{\mathrm{t}}[\mathbf{c}] \mathcal{B} \mathbf{u} \mathrm{d} \Omega+\int_{\Gamma_{\mathrm{sa}}} \mathbf{w} \cdot \mathbf{n}_{\mathrm{s}} p \mathrm{~d} \Gamma=\int_{\Gamma_{\mathrm{P}}^{\mathrm{s}}} p_{\mathrm{n}} \mathbf{w} \cdot \mathbf{n}_{\mathrm{S}} \mathrm{d} \Gamma, \\
\int_{\Omega_{\mathrm{a}}} \frac{1}{c^{2}} w \ddot{p} \mathrm{~d} \Omega+\int_{\Omega_{\mathrm{a}}} \nabla w \cdot \nabla p \mathrm{~d} \Omega+\int_{\Gamma_{\mathrm{abc}}^{\mathrm{a}}} w \frac{1}{c} \frac{\partial p}{\partial t} \mathrm{~d} \Gamma-\int_{\Gamma_{\mathrm{sa}}} w \rho_{\mathrm{a}} \mathbf{n}_{\mathrm{s}} \cdot \frac{\partial^{2} \mathbf{u}}{\partial t^{2}} \mathrm{~d} \Gamma=0 .
\end{array}
$$

After discretizing the system (10), (11) with Lagrangian finite elements, we arrive at the 
semi-discrete Galerkin formulation

$$
\left(\begin{array}{cc}
\mathbf{M}_{\mathbf{u}} & 0 \\
-\mathbf{M}_{\mathbf{u} p} & \mathbf{M}_{p}
\end{array}\right)\left(\begin{array}{l}
\underline{\ddot{\mathbf{u}}} \\
\ddot{p}
\end{array}\right)+\left(\begin{array}{cc}
\mathbf{C}_{\mathbf{u}} & 0 \\
0 & \mathbf{C}_{p}
\end{array}\right)\left(\begin{array}{l}
\underline{\dot{\mathbf{u}}} \\
\dot{\underline{p}}
\end{array}\right)+\left(\begin{array}{cc}
\mathbf{K}_{\mathbf{u}} & \mathbf{K}_{p \mathbf{u}} \\
0 & \mathbf{K}_{p}
\end{array}\right)\left(\begin{array}{l}
\underline{\mathbf{u}} \\
\underline{p}
\end{array}\right)=\left(\begin{array}{l}
\underline{\mathbf{f}} \\
\mathbf{0}
\end{array}\right) .
$$

Now, since in most cases the discretization within the solid has to be much finer than the one needed for the acoustic wave propagation in the fluid, we will use nonmatching grid techniques to allow different grids for mechanics and acoustics (see Fig. 3(b)). Therewith, the coupling matrix $\mathbf{K}_{p \mathbf{u}}$ computes element-wise as

$$
\mathbf{K}_{p \mathbf{u}}=\bigwedge_{e=1}^{n_{\text {isec }}} \mathbf{k}^{e} ; \mathbf{k}^{e}=\left[k_{a b}\right] ; \quad k_{a b}=\int_{\Gamma_{e}} \mathbf{N}_{a}^{j} \cdot \mathbf{n} N_{b}^{k} \mathrm{~d} \Gamma .
$$

Here $\wedge$ is the element-wise FE assembly operator and $n_{\text {isec }}$ is the number of intersection elements on the interface since the surface integrals over $\Gamma_{\mathrm{sa}}$ have to be evaluated with respect to different grids as shown in Fig. 3(b). This makes the introduction of grid intersection operators necessary $[9,10]$.

The finite element basis functions $N_{a}^{j}$ and $N_{b}^{k}$ in (13) denote the traces of the FE basis on the mechanical and on the acoustic side of the interface. Basically the coupling matrix $\mathbf{M}_{\mathbf{u} p}$ is linked to the matrix $\mathbf{K}_{p \mathbf{u}}$ through the mean density of the acoustic fluid by the equation $\mathbf{M}_{\mathbf{u} p}=\rho_{0} \mathbf{K}_{p \mathbf{u}}^{t}$. The damping matrix $\mathbf{C}_{\mathbf{u}}$ is due to the introduction of Rayleigh damping. It computes as $\mathbf{C}_{\mathbf{u}}=\alpha_{\mathrm{M}} \mathbf{M}_{\mathbf{u}}+\alpha_{\mathrm{K}} \mathbf{K}_{\mathbf{u}}$. The coefficients $\alpha_{\mathrm{M}}$ and $\alpha_{\mathrm{K}}$ are related to the modal loss factor, $\tan \delta_{i}$, via the equation $\tan \delta_{i}=\left(\alpha_{\mathrm{M}}+\alpha_{\mathrm{K}} \omega_{i}^{2}\right) / \omega_{i}$ with $\omega_{i}$ being the $i$-th eigenfrequency of the mechanical system. For the computation of the coefficients from measured data we refer to $[5,11]$. The acoustic damping matrix $\mathbf{C}_{p}$ is due to the incorporation of the absorbing boundary condition on $\Gamma_{\mathrm{abc}}^{\mathrm{a}}$. The time discretization is performed by applying the standard implicit Newmark time stepping scheme [11], e.g., using the time stepping parameters $\beta=0.25$ and $\gamma=0.5$.

\subsection{Application: Active Damping by Piezoelectric Patches}

In many engineering applications vibrations are responsible for the generation of acoustic noise. Especially slender or thin-walled structures with a large surface exhibit these effects. A modern way of controlling vibrations is to attach piezoelectric patches to membrane/plate like structures, which can measure the deformation and which act against these deformations, when the patches are loaded by adequate voltage signals. These enhanced devices are so-called smart materials.

It is our goal to simulate such devices with the flexibility to arbitrarily place the piezoelectric patches on the plate structure without the need to re-mesh the computational domain. Our considered setup is displayed in Fig. 4. Therewith, we can efficiently investigate the optimal position of the piezoelectric actuators to minimize mechanical vibrations. This flexibility is provided by the Mortar FEM, which allows to couple computational grids with different mesh sizes. Similar to the acoustic-acoustic coupling (see Sec. 4.1) we are now concerned with a mechanical-mechanical coupling between supporting plate and piezoelectric 
patch. Therewith, we force the continuity of the mechanical displacement in a weak sense

$$
\int_{\Gamma_{I}}\left(\mathbf{u}_{1}-\mathbf{u}_{2}\right) \boldsymbol{\mu} \mathrm{d} \Gamma=0
$$

with $\boldsymbol{\mu}$ from a suitable Lagrange multiplier space $M^{n_{\mathrm{d}}}$ (cf. $\left.[9,12,13]\right)$. In addition, we apply a strong condition on the normal stresses by introducing the Lagrange multiplier (LM) $\boldsymbol{\lambda}$

$$
\lambda=-\left[\boldsymbol{\sigma}_{1}\right] \cdot \mathbf{n}=-\left[\boldsymbol{\sigma}_{2}\right] \cdot \mathbf{n} .
$$

The piezoelectric effect is modeled by the standard PDE for mechanics (see Sec. 2.1) and the PDE for electrostatics, using the scalar electric potential $V$ and the piezoelectric constitutive law coupling the mechanical and electric physical quantities (see, e.g., [5]).

Furthermore, since the discretization requirements for the supporting plate and the ambient fluid are very different, we will also use a nonmatching grid along the common interface between the plate and the ambient acoustic computational domain. To strengthen this point we consider the acoustic wave length in air at $100 \mathrm{~Hz}$, which is $\lambda=3.4 \mathrm{~m}$. This would permit the usage of linear finite elements with an edge length of about $\lambda / 20=17 \mathrm{~cm}$. The metal plate which excites the acoustic wave is however of rectangular dimensions $50 \mathrm{~cm}$ by $30 \mathrm{~cm}$ by $1 \mathrm{~mm}$. Therewith, we discretize the plate as a 3D solid and use 2nd order finite element basis functions with a special selected reduced integration technique to avoid locking [14]. To accurately simulate the mechanical deformations of this plate we need to apply a mesh size of about $5 \mathrm{~mm}$ in plane and $1 \mathrm{~mm}$ in thickness direction. This means that the acoustic mesh size (linear finite elements) may be one order of magnitude (34 times at $100 \mathrm{~Hz}$ ) larger in plane direction than the mesh size (2nd order finite elements) for the mechanical computation.

Now, the overall formulation in the frequency domain (harmonic analysis) results in the following complex algebraic system of equations

$$
\left(\begin{array}{ccccc}
\mathbf{K}_{\boldsymbol{u}_{1}}^{*} & 0 & \mathbf{D}^{t} & \mathbf{K}_{\boldsymbol{u}_{1} V} & 0 \\
0 & \mathbf{K}_{\boldsymbol{u}_{2}}^{*} & \mathbf{M}^{t} & 0 & \mathbf{K}_{\boldsymbol{u}_{2} p} \\
\mathbf{D} & \mathbf{M} & 0 & 0 & 0 \\
\mathbf{K}_{\boldsymbol{u}_{1} V}^{t} & 0 & 0 & -\mathbf{K}_{V V} & 0 \\
0 & -\omega^{2} \mathbf{M}_{p \boldsymbol{u}_{2}} & 0 & 0 & \mathbf{K}_{p}^{*}
\end{array}\right)\left(\begin{array}{c}
\frac{\hat{\boldsymbol{u}}_{1}}{\hat{\boldsymbol{u}}_{2}} \\
\underline{\hat{\hat{\lambda}}} \\
\underline{\hat{V}} \\
\underline{\hat{p}}
\end{array}\right)=\left(\begin{array}{c}
0 \\
0 \\
0 \\
\underline{\hat{f}_{\mathrm{e}}} \\
0
\end{array}\right)
$$

with

$$
\begin{aligned}
& \mathbf{K}_{\boldsymbol{u}_{1}}^{*}=-\mathbf{K}_{\boldsymbol{u}_{1}}+j \omega \mathbf{C}_{\boldsymbol{u}_{1}}-\omega^{2} \mathbf{M}_{\boldsymbol{u}_{1}} ; \quad \mathbf{K}_{p}^{*}=\mathbf{K}_{p}-\omega^{2} \mathbf{M}_{p} \\
& \mathbf{K}_{\boldsymbol{u}_{2}}^{*}=\mathbf{K}_{\boldsymbol{u}_{2}}+j \omega \mathbf{C}_{\boldsymbol{u}_{2}}-\omega^{2} \mathbf{M}_{\boldsymbol{u}_{2}} .
\end{aligned}
$$

The two matrices $\mathbf{D}, \mathbf{M}$ care for the mechanical - mechanical nonmatching grid interface (supporting plate and piezoelectric patch), and the two matrices $\mathbf{K}_{\boldsymbol{u}_{2} p}, \mathbf{M}_{p \boldsymbol{u}_{2}}$ for the mechanical-acoustic nonconforming interface (supporting plate and ambient fluid). 


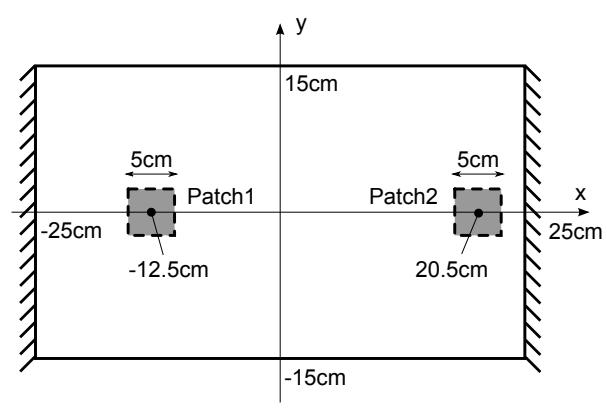

(a) Plate from top.

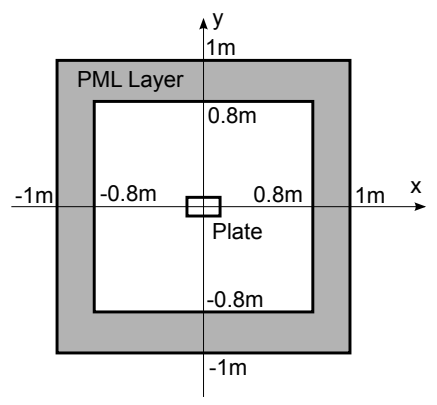

(b) Acoustic domain from top.

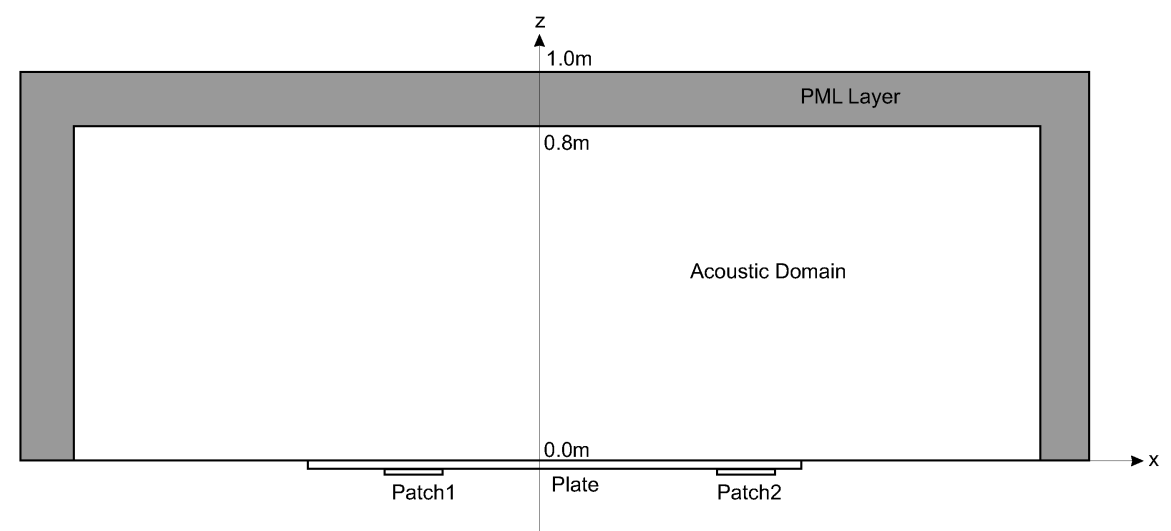

(c) Computational domain at the front.

Fig. 4. Setup of computational domain (for display reasons not at scale).

The geometry for our setup consists of a metal plate of thickness $1 \mathrm{~mm}$ with two attached piezoelectric patches as depicted in Fig. 4. The thickness of both patches is also $1 \mathrm{~mm}$. They are attached below the plate at the given positions. The top surface of the plate coincides with $\mathrm{z}=0$. The bottom surface of the acoustic domain also coincides with the plane $\mathrm{z}=0$. The acoustic propagation domain extends $80 \mathrm{~cm}$ in z-direction. An additional $20 \mathrm{~cm}$ layer for applying a perfectly matched layer (cf. [15]) to simulate free field radiation is added on top of the propagation domain.

As materials we choose aluminum for the plate, lead zirconate titanate (PZT-4) for both patches and air for the acoustic propagation region. For the electrostatic field we apply homogeneous Dirichlet conditions on the bottom electrodes and inhomogeneous Dirichlet conditions on the top electrodes to prescribe the electric potential. Homogenous Neumann conditions are used on the rest of the faces. The plate is mechanically fixed for all degrees of freedom on its left and right boundaries and may move freely anywhere else.

In a first step, we just excite patch 1 by applying a voltage load at $80 \mathrm{~Hz}$. Figure 5(a) displays the mesh in the vicinity of patch 2 to demonstrate the large difference between the mesh size in the plate and the ambient fluid. The computed acoustic sound pressure on a 


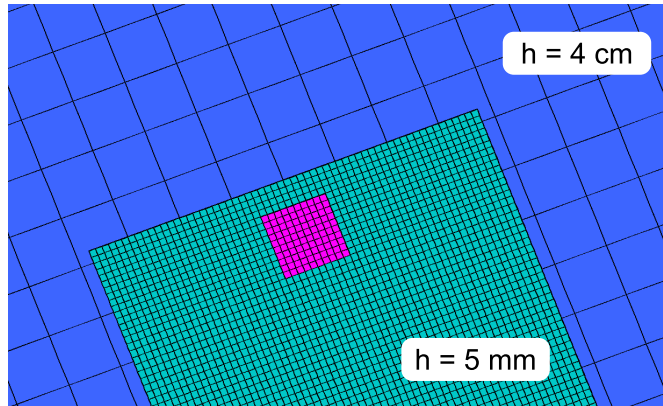

(a) Nonmatching Grid.

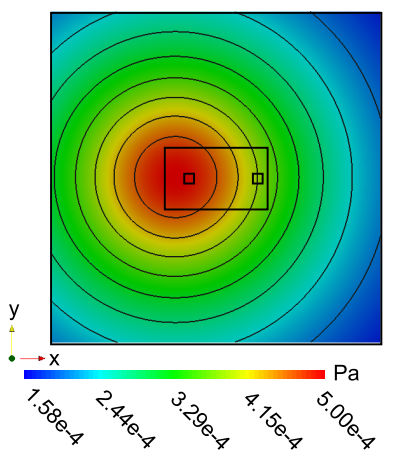

(b) Amplitude of sound pressure at $\mathrm{z}=50 \mathrm{~cm}($ at $80 \mathrm{~Hz})$.

Fig. 5. Nonmatching grid and acoustic pressure at $80 \mathrm{~Hz}$

plane $50 \mathrm{~cm}$ above the plate is shown in Fig. $5(\mathrm{~b}))$.

In a second step, we apply the inverse voltage $\hat{U}=-10 \mathrm{~V}$ to patch 2 and study the amount of reduction for the mechanical displacement (see Fig. 6). For the investigated

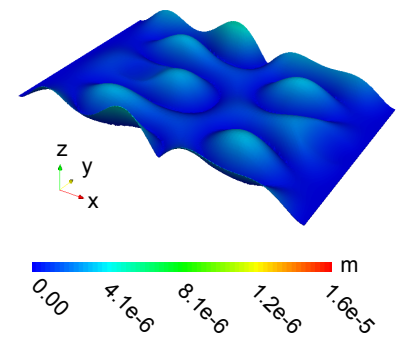

(a) Original Configuration.

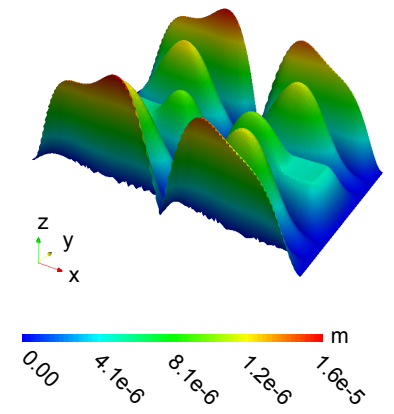

(b) Patch2 $3 \mathrm{~cm}$ closer to (c) Patch1.

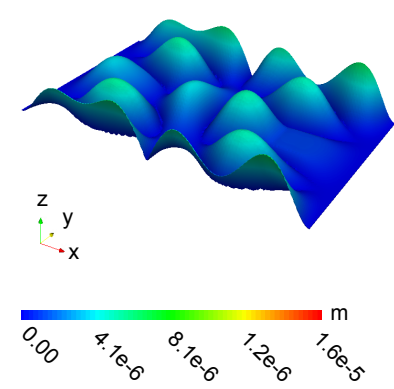

$$
\text { Patch1. }
$$

Fig. 6. Mechanical displacements on plate for different positions of Patch2.

setup, the position of patch 2 is already a quite optimal one. Changing its position just by some small amount, as shown in Figure 6, strongly increases the mechanical vibration amplitude and furthermore the radiated sound as demonstrated in Fig. 7.

\section{High-Intensity Focused Ultrasound}

High-power ultrasound sources have found their way into a wide variety of applications, ranging from medical ultrasound, like lithotripsy or HIFU-therapy (High-Intensity Focused Ultrasound) to ultrasonic cleaning or welding and sonochemistry [16]. In contrast to ultrasonic applications with low amplitude pressure waves non-linear effects like sawtooth 


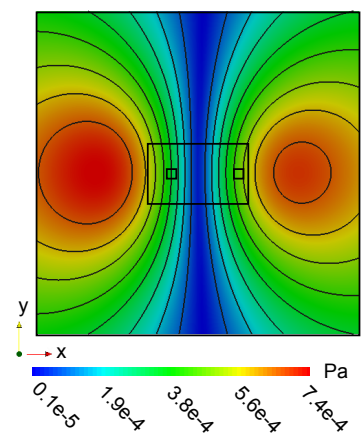

(a) Original Configuration.

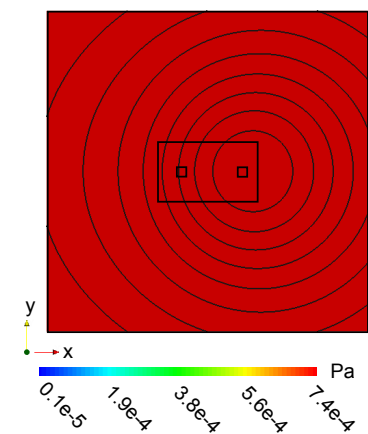

(b) Patch2 $3 \mathrm{~cm}$ closer to (c) Patch1.

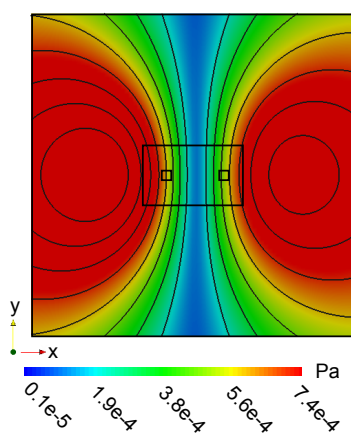

(c) Patch2 $5 \mathrm{~cm}$ closer to Patch1.

Fig. 7. Acoustic amplitude field at $\mathrm{z}=50 \mathrm{~cm}$ for different positions of Patch2.

and shock formation occur within high-power ultrasound. Appropriate numerical simulation tools are required to speed up their design process. These have to include the electromechanical and the fluid-solid coupling and must be capable to compute the propagation of finite amplitude waves through lossy and compressible media.

A quite general approach, which fulfills these requirements is Kuznetsov's equation [17], which is a second-order approximation for viscous heat-conducting fluids

$$
c^{2} \Delta \psi-\frac{\partial^{2} \psi}{\partial t^{2}}=-\frac{\partial}{\partial t}\left(b \Delta \psi+\frac{1}{c^{2}} \frac{B}{2 A}\left(\frac{\partial \psi}{\partial t}\right)^{2}+\nabla \psi \cdot \nabla \psi\right) .
$$

In (16) $b$ denotes the diffusivity of sound and $B / A$ the parameter of non-linearity. Besides Kuznetsov's equation, the following three partial differential equations for modeling nonlinear wave propagation are widely used (see e.g., [18]):

- Burger's equation:

$$
\frac{\partial p^{\prime}}{\partial x}-\frac{b}{2 c^{3}} \frac{\partial^{2} p^{\prime}}{\partial \tau^{2}}=\frac{\beta_{a}}{\rho_{0} c^{3}} p \frac{\partial p^{\prime}}{\partial \tau}
$$

with $\tau=(t-x / c)$ the retarded time, $b$ the diffusivity of sound and $\beta_{a}=1+B / 2 A$ the coefficient of non-linearity. Burger's equation allows the investigation of the combined effect of dissipation and non-linearity on progressive one-dimensional plane waves.

- Westervelt equation:

$$
\nabla^{2} p^{\prime}-\frac{1}{c^{2}} \frac{\partial^{2} p^{\prime}}{\partial t^{2}}+\frac{b}{c^{4}} \frac{\partial^{3} p^{\prime}}{\partial t^{3}}=-\frac{\beta_{a}}{\rho_{0} c^{4}} \frac{\partial^{2} p^{\prime 2}}{\partial t^{2}}
$$

with $b$ the diffusivity of sound. This partial differential equation can describe the propagation of plane waves including the non-linear effects as well as dissipation. This means that 
we can apply this equation, when cumulative non-linear effects dominate local non-linear effects, e.g., in cases where the distance of propagation is larger than one wavelength. The approximation is not appropriate for the simulation of standing waves.

- Khokhlov-Zabolotskaya-Kuznetsov (KZK) equation:

$$
\frac{\partial^{2} p^{\prime}}{\partial x \partial t}-\frac{c}{2} \nabla_{\perp}^{2} p^{\prime}-\frac{b}{2 c^{3}} \frac{\partial^{3} p^{\prime}}{\partial t^{3}}=\frac{\beta_{a}}{2 \rho_{0} c^{3}} \frac{\partial^{2} p^{\prime 2}}{\partial t^{2}}
$$

with $\beta_{a}=1+B / 2 A$ the coefficient of non-linearity, $b$ the diffusivity of sound and $\nabla_{\perp}$ the nabla operator including only partial derivatives with respect to the transversal coordinates. The simplification w.r.t. to Kuznetsov's equation is given by modeling only directional wave propagation fulfilling

$$
\frac{k_{\perp}}{k_{x}} \ll 1,
$$

with $k_{\perp}$ the wave number in the transversal direction and $k_{x}$ the wave number in the direction of propagation. This approximation is mainly used for investigation of diffraction, non-linearity and dissipative effects in directional sound beams which occur, e.g., in medical ultrasound, acoustic microscopy or non-destructive testing.

Besides the correct modeling of the non-linearities within the wave propagation, the process of heat transfer in biological tissue has to be modeled. A general approach is given by the equation known either as the bio-heat equation or as the Pennes equation [19]. Often, the thermal model of interest can be derived from this equation by a truncation of the blood perfusion and metabolic activity terms. Thus the problem of heat transfer for the temperature $T$ reads as

$$
\rho(T) c_{\nu} \frac{\partial T}{\partial t}=\kappa \Delta T+\langle q\rangle
$$

with the density $\rho$, the specific heat capacity $c_{\nu}$, the specific heat conductivity $\kappa$ and the temporal averaged heat source $\langle q\rangle$. The acoustic source term $q$ is the total acoustic energy absorbed and converted to heat, which is defined over the acoustic intensity $\mathbf{I}$ as

$$
q=-\nabla \cdot \mathbf{I} \text { with } \mathbf{I}=p^{\prime} \mathbf{v}^{\prime} .
$$

In terms of the acoustic potential the source term computes as

$$
q=\rho_{0}\left(\nabla \frac{\partial \psi}{\partial t} \cdot \nabla \psi+\frac{\partial \psi}{\partial t} \Delta \psi\right)
$$

where $\rho_{0}$ is the mean density. This source term was firstly obtained by Nyborg [20] and later in [21], and is valid for arbitrary wave propagation. We remark that this temporal averaging must be performed over a sufficiently long time interval within which the wave does not change its general character. 


\subsection{FE Formulation for High Intensity Focused Ultrasound}

In the following, we will summerize the main steps for the numerical solution of Kuznetsov's equation by an enhanced FE formulation. The strong formulation for the non-linear wave equation by (16) reads as: Given

$$
\psi_{0}, \dot{\psi}_{0}: \Omega_{\mathrm{a}} \rightarrow \mathbb{R} \text { and } f, c, B / A, b: \Omega_{\mathrm{a}} \rightarrow \mathbb{R}
$$

find the scalar acoustic potential $\psi: \overline{\Omega_{\mathrm{a}}} \times[0, T] \rightarrow \mathbb{R}$ such that

$$
\frac{1}{c^{2}} \frac{\partial^{2} \psi}{\partial t^{2}}-\Delta \psi=f+\frac{1}{c^{2}} \frac{\partial}{\partial t}\left(b(\Delta \psi)+\frac{B / A}{2 c^{2}}\left(\frac{\partial \psi}{\partial t}\right)^{2}+(\nabla \psi)^{2}\right)
$$

with Dirichlet and Neumann boundary conditions of the form

$$
\psi=\psi_{\mathrm{D}} \quad \text { on } \quad \Gamma_{\mathrm{D}}^{\mathrm{a}} \times(0, T) ; \quad \frac{\partial \psi}{\partial \mathbf{n}}=\psi_{\mathrm{N}} \quad \text { on } \quad \Gamma_{\mathrm{N}}^{\mathrm{a}} \times(0, T),
$$

and initial conditions

$$
\psi(\mathbf{x}, 0)=\psi_{0}, \dot{\psi}^{\prime}(\mathbf{x}, 0)=\dot{\psi}_{0}, \mathbf{x} \in \Omega_{\mathrm{a}} .
$$

For simplicity we set all boundary conditions as well as initial conditions to zero. Now, to obtain the variational formulation, we multiply (21) by an appropriate test function $w$ and perform an integration by parts for the Laplace term on the left- and right-hand sides. Thus, the variational formulation reads as follows: Find $\psi \in V=\left\{\varphi \in H^{1}\left(\Omega_{\mathrm{a}}\right) \mid \varphi=p_{\mathrm{D}}\right.$ on $\left.\Gamma_{\mathrm{D}}^{\mathrm{a}}\right\}$ such that

$$
\begin{aligned}
\int_{\Omega} \frac{1}{c^{2}} w \ddot{\psi} \mathrm{d} \Omega+\int_{\Omega}(\boldsymbol{\nabla} w) \cdot(\boldsymbol{\nabla} \psi) \mathrm{d} \Omega & =\int_{\Omega} w f \mathrm{~d} \Omega-\int_{\Omega} \boldsymbol{\nabla}\left(\frac{b}{c^{2}} w\right) \cdot(\boldsymbol{\nabla} \dot{\psi}) \mathrm{d} \Omega \\
& +\int_{\Omega} \frac{2}{c^{4}} \frac{B}{2 A} w \dot{\psi} \ddot{\psi} \mathrm{d} \Omega+\int_{\Omega} w \boldsymbol{\nabla}\left(\frac{2}{c^{2}} \psi\right) \cdot(\boldsymbol{\nabla} \dot{\psi}) \mathrm{d} \Omega
\end{aligned}
$$

for all $w \in W=\left\{q \in H^{1}\left(\Omega_{\mathrm{a}}\right) \mid q=0\right.$ on $\left.\Gamma_{\mathrm{D}}^{\mathrm{a}}\right\}$. Applying standard Lagrangian finite elements, we arrive at

$$
\mathbf{M}_{\psi} \underline{\ddot{\psi}}+\mathbf{K}_{\psi} \underline{\psi}+\mathbf{C}_{\psi} \dot{\underline{\psi}}-\mathbf{N}_{\psi}^{1}(\dot{\psi}) \underline{\ddot{\psi}}-\mathbf{N}_{\psi}^{2}(\psi) \underline{\dot{\psi}}=\underline{f} .
$$

The time discretization is performed by an implicit Newmark algorithm with time stepping parameters $\beta=0.25$ and $\gamma=0.5$. Since (23) is a non-linear equation, we have to apply an iterative scheme. By shifting all non-linearities to the right-hand side of the equation system, we arrive at the following scheme:

(1) Perform predictor step:

$$
\begin{aligned}
& \underline{\tilde{\psi}}=\underline{\psi}_{n}+\Delta t \underline{\dot{\psi}}_{n}+\frac{\Delta t^{2}}{2}(1-2 \beta) \underline{\ddot{\psi}}_{n}=\underline{\psi}_{n+1}^{k} \\
& \underline{\tilde{\dot{\psi}}}=\underline{\dot{\psi}}_{n}+(1-\gamma) \Delta t \underline{\ddot{\psi}}_{n}=\underline{\dot{\psi}}_{n+1}^{k} .
\end{aligned}
$$


(2) Solve algebraic system of equations:

$$
\begin{aligned}
\mathbf{M}_{\psi}^{*} \underline{\ddot{\psi}}_{n+1}^{k+1} & =\underline{f}_{n+1} \\
& -\mathbf{K}_{\psi} \underline{\tilde{\psi}}-\mathbf{C}_{\psi} \underline{\tilde{\dot{\psi}}}+\mathbf{N}_{\psi}^{1}\left(\underline{\dot{\psi}}_{n+1}^{k}\right) \underline{\ddot{\psi}}_{n+1}^{k}+\mathbf{N}_{\psi}^{2}\left(\underline{\psi}_{n+1}^{k}\right) \underline{\dot{\psi}}_{n+1}^{k} \\
\mathbf{M}_{\psi}^{*} & =\mathbf{M}_{\psi}+\gamma \Delta t \mathbf{C}_{\psi}+\beta \Delta t^{2} \mathbf{K}_{\psi} .
\end{aligned}
$$

(3) Perform corrector step:

$$
\begin{aligned}
& \underline{\psi}_{n+1}^{k+1}=\underline{\tilde{\psi}}+\beta \Delta t^{2} \ddot{\ddot{\psi}}_{n+1}^{k+1} \\
& \underline{\dot{\psi}}_{n+1}^{k+1}=\underline{\tilde{\dot{\psi}}}^{h}+\gamma \Delta t \underline{\ddot{\psi}}_{n+1}^{k+1} .
\end{aligned}
$$

(4) Test convergence:

$$
\frac{\left\|\underline{\ddot{\psi}}_{n+1}^{k+1}-\ddot{\ddot{\psi}}_{n+1}^{k}\right\|_{2}}{\left\|\ddot{\psi}_{n+1}^{k+1}\right\|_{2}} \leq \delta_{\psi}\left\{\begin{array}{l}
\text { fulfilled } \quad: \text { perform next time step } \\
\text { not fulfilled }: k:=k+1 \text { goto step } 2
\end{array} .\right.
$$

In order to demonstrate the non-linearity, we consider in a first example a simple acoustic channel setup as displayed in Fig. 8. The vibrating body is excited by a sine-burst with an

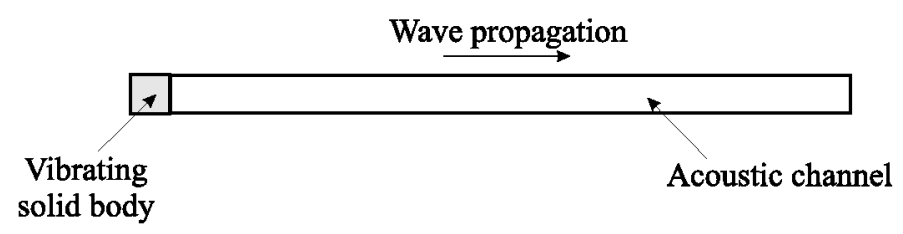

Fig. 8. Simple computational setup and excitation signal to demonstrate the generation of non-linear waves.

amplitude of $100 \mu \mathrm{m}$ at $100 \mathrm{kHz}$. For the medium we use water $(c=1500 \mathrm{~m} / \mathrm{s})$ with a parameter of non-linearity $B / A$ equal to 5 and a diffusivity of sound value $b=6 \cdot 10^{-9} \mathrm{~m}^{2} / \mathrm{s}$. We choose a FE mesh with 250 finite elements per fundamental wavelength $\left(\lambda_{\text {fundamental }}=\right.$ $\left.c_{\text {water }} / f_{\text {fundamental }}=1500 / 10^{5}\right)$ and set the time step size to $20 \mathrm{~ns}$, which corresponds to 500 time samples per fundamental time period $\left(T_{\text {fundamental }}=1 / f_{\text {fundamental }}\right)$. The fine spatial and time discretization is necessary to correctly resolve the higher harmonics, which arise due to the non-linearity. Figure 9 shows the time signal and frequency spectra exactly at the shock formation distance (for the analytical solution we refer to [22]). As the wave further propagates, its shape will become a saw-tooth as displayed in Fig. 10 (for the analytical solution at this distance we refer to [22]).

In a second example, we want to demonstrate for a coupled acoustic-thermal simulation the strong difference between a realistic model (uses Kuznetsov's equation with temperature dependent speed of sound) and a quite simple model (uses linear wave equation with constant speed of sound). Therewith, we introduce a computational domain 


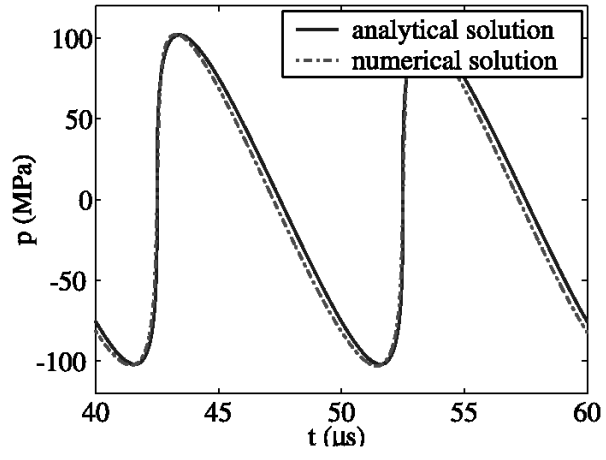

(a) Time signal.

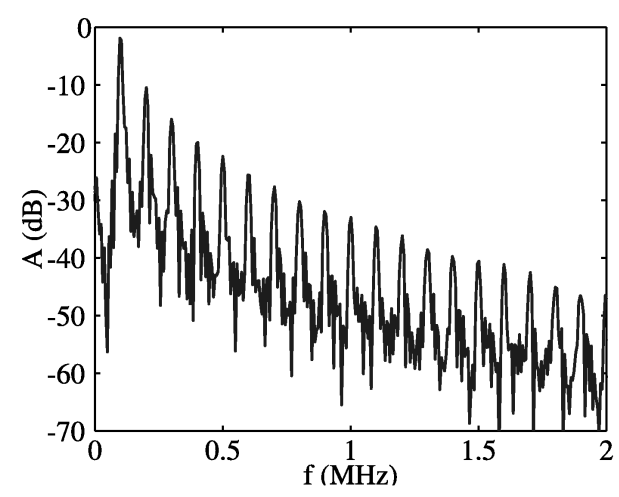

(b) Frequency spectrum.

Fig. 9. Time signal and frequency spectrum of the non-linear wave at the shock formation distance.

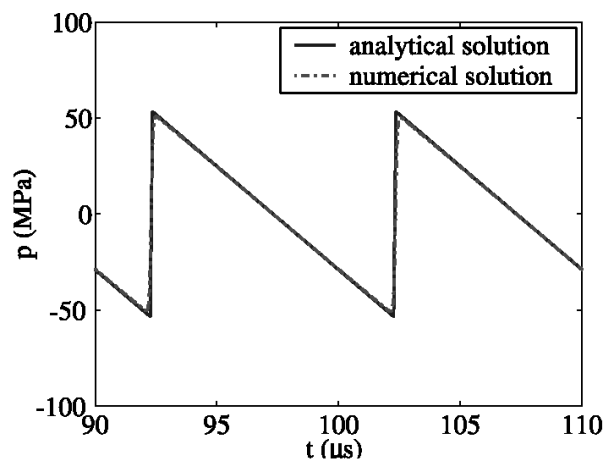

(a) Five times the shock formation distance.

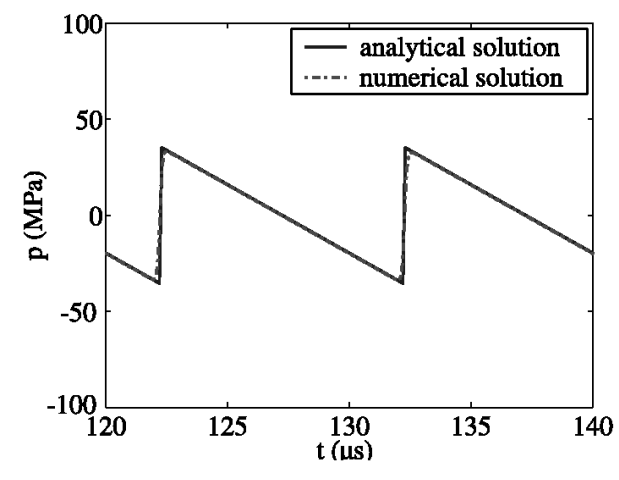

(b) Eight times the shock formation distance.

Fig. 10. Time signal of the non-linear wave at five and eight times the shock formation distance.

$\Omega=\Omega_{\mathrm{F}} \cup \Omega_{\mathrm{T}}$ which is divided into two sub-domains $\Omega_{\mathrm{F}}=[0,0.01 \mathrm{~m}] \times[0,0.005 \mathrm{~m}]$ and $\Omega_{\mathrm{T}}=[0,0.01 \mathrm{~m}] \times[0.005 \mathrm{~m}, 0.025 \mathrm{~m}]$ (see Fig. 11 ). These two subregions represent a waterliver system with temperature dependencies for the speed of sound as well as density (for details see [23]). The wave transducer with an aperture size of $20 \mathrm{~mm}$ located at the bottom of the domain emits ultrasound waves at a frequency of $1 \mathrm{MHz}$ traveling from bottom to top. For simplicity, we do not model the actual transducer (in most cases a piezoelectric transducer), but instead model the transducer by the following inhomogeneous Neumann boundary condition

$$
\frac{\partial \psi}{\partial \mathbf{n}}=-\sin \left(2 \pi 10^{6} t\right) \quad \text { on } \Gamma_{E} \times\left[0, t_{n}\right] .
$$

Furthermore, we set second order absorbing boundary conditions for the acoustic scalar potential on all other boundaries of the computational domain [23]. The temperature field 


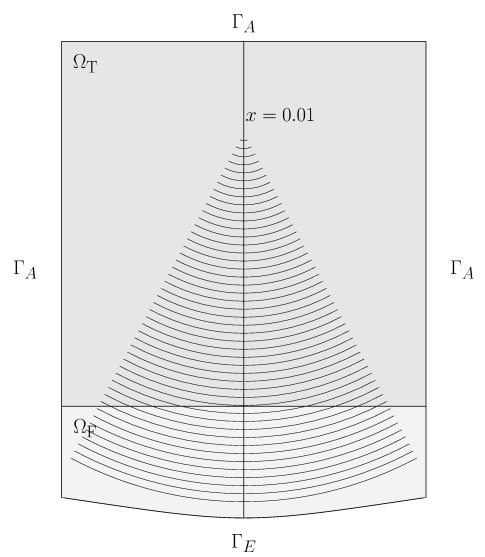

Fig. 11. The computational setup for the ultrasonic heating problem.

is set to $293.15 \mathrm{~K}$ on all boundaries. For the sake of convenience in exposition the acoustic potential/pressure and the temperature field are normalized to their maximum values. From

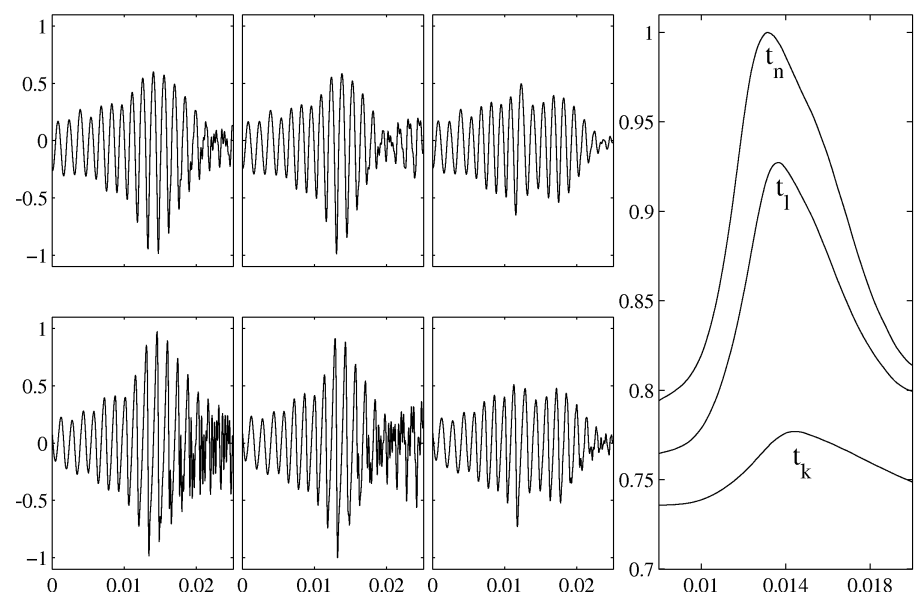

Fig. 12. The evolution of the acoustic potential/pressure (upper/lower row) profile at time moments $t_{k}, t_{l}$, $t_{n}$ with $t_{k}<t_{l}<t_{n}$ (from left to right) and the temperature focus (the rightmost picture) for the realistic model (Kuznetsov equation with temperature dependent speed of sound). The horizontal axis represents the line $x=0.01$ in the computational domain $\Omega$ (see Fig. 11).

Fig. 12 one can see how the acoustic potential/pressure and temperature peaks moves in the direction of the acoustic source that is caused by the thermo-acoustic lensing effect. However, as displayed in Fig. 13 the simple model does not show any thermo-acoustic lensing effect and furthermore, the temperature increase is quite smaller. 


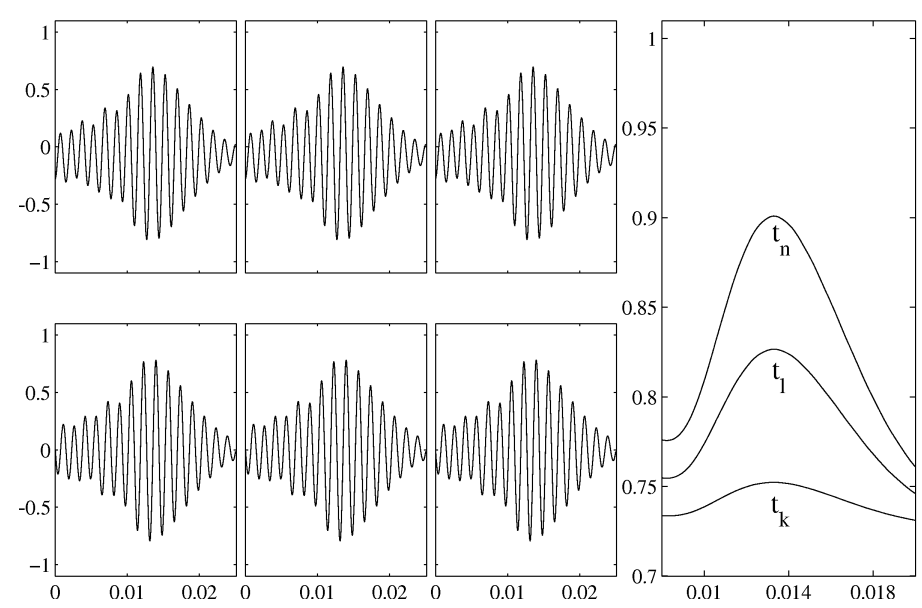

Fig. 13. The evolution of the acoustic potential/pressure (upper/lower row) profile at time moments $t_{k}, t_{l}$, $t_{n}$ (from left to right) and the temperature focus (the rightmost picture) for the simple model (linear wave equation with constant speed of sound). The horizontal axis represents the line $x=0.01$ in the computational domain $\Omega$ (see Fig. 11).

\subsection{Application: High-Intensity Focused Ultrasound Sources}

The principle setup of an acoustic power source based on the piezoelectric effect is shown in Fig. 14(a). Due to the geometric focusing of the lens, high acoustic intensity can be achieved in the focus region. The piezoelectric transducer has a diameter of $60 \mathrm{~mm}$ and the radius of

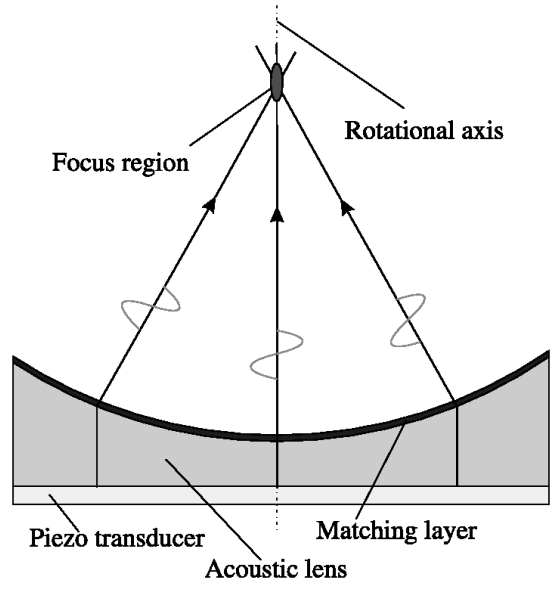

(a) Principle setup of high-power ultrasound source.

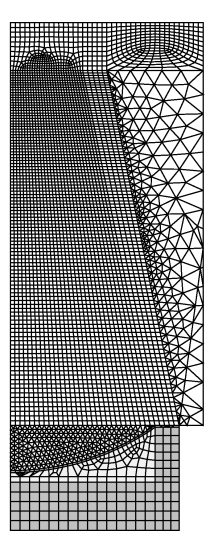

(b) Finite element mesh (for display reasons, just a coarse mesh is shown).

Fig. 14. Setup of a high-power ultrasound source and corresponding FE mesh (axisymmetric model). 
curvature of the lense is $55 \mathrm{~mm}$, which results in a focal distance of $70 \mathrm{~mm}$. The operating frequency of the transducer is $1.7 \mathrm{MHz}$.

At the starting point in our investigations impedance calculations have been performed for the piezoelectric disc transducer. This was mainly performed to obtain all necessary and unknown material parameters. By applying an inverse scheme, in which we fully simulate the transducer and automatically adjust the material parameters to fit measured and computed electric impedance, we obtain the dielectric, mechanical and piezoelectric material values [24-26]. The simulated and the measured electric impedance of the piezoelectric transducer

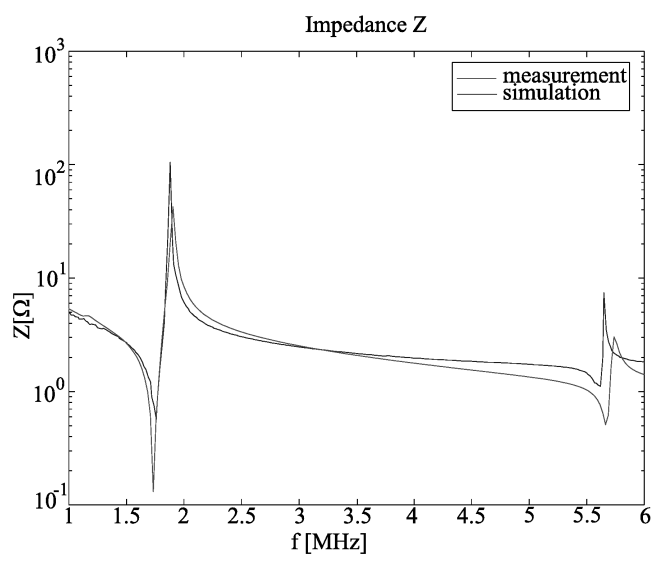

(a) Impedance of the M453 piezo transducer in air.

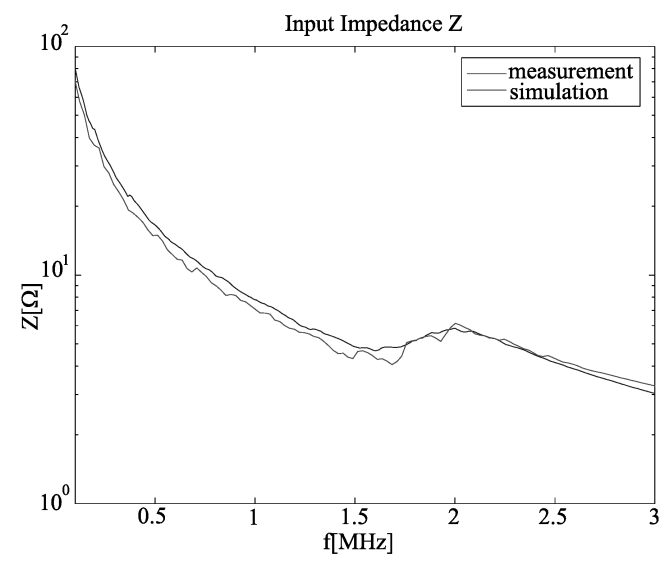

(b) Electrical input impedance of the HIFU source loaded with water.

Fig. 15. Two typical acoustic field computations.

is displayed in Fig. 15(a). Due to good agreement over a wide frequency range, we can trust the fitted material parameters. Next, the impedance of the whole HIFU source was simulated with water load. The computed electric impedance in water is displayed in Fig. $15(\mathrm{~b})$.

In the numerical simulation of the HIFU source, the piezoelectric and the fluid-solid coupling as well as the non-linear wave propagation in the fluid is taken into account. Therefore, an axi-symmetric finite element model has been setup in which the piezoelectric transducer, the lens, the matching layer and the water has been discretized (see Fig. 14(b)).

The FE model consists of about one million finite elements just in the acoustic computational domain. Therewith, at least 8 linear finite elements per wavelength $\lambda$ are guaranteed for the first 4 harmonics. For the excitation of the piezoelectric transducer a sine burst at $1.7 \mathrm{MHz}$ with a maximal amplitude of $U=133 V_{\mathrm{pp}}$ has been chosen. The simulation results were observed at several points on the rotational axis between the source and the focus region. A transient analyses has been performed for 13.500 time steps with a time step size of $4 \mathrm{~ns}$. It should be noted that also for the higher excitation voltage the piezoelectric transducer is still in the linear region. Therefore, any distortions in the pressure signal 
arise from non-linear effects in the fluid only. The comparison of measurements (see [27]) with simulation results is shown in Figs. 16(a) and 16(b). The non-linear distortion of the sine wave due to the generation of higher harmonics is clearly visible. In the simulations a damping value for the fluid of $0.22 \mathrm{~dB} / \mathrm{MHz}^{2} \mathrm{~m}$ has been used.

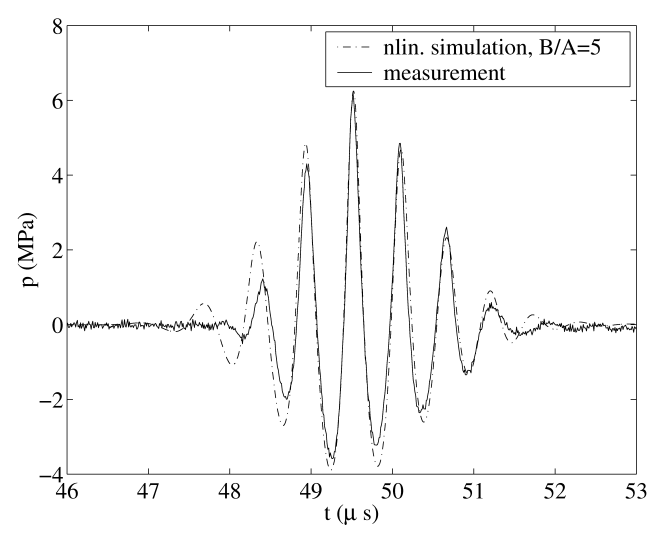

(a) Pressure pulse signal for high-intensity measurement and non-linear simulation at the focal distance.

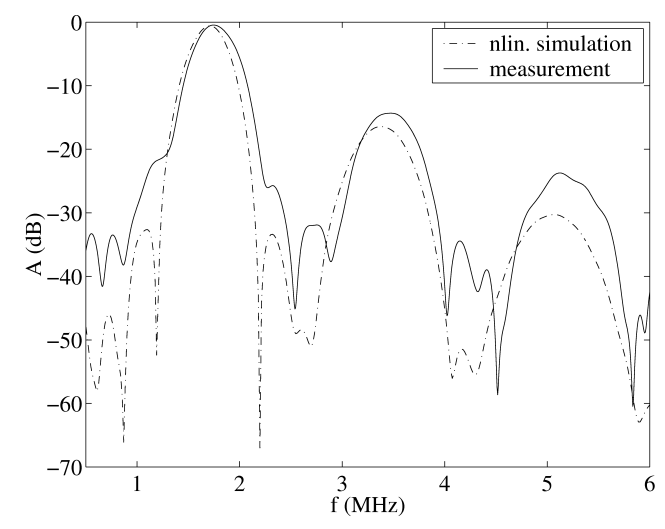

(b) Frequency spectrum of pressure pulse for high-intensity measurement and non-linear simulation at the focal distance.

Fig. 16. Time signal and frequency spectrum for the non-linear simulation compared to measured data.

In a second example, we will discuss the numerical computation of a high-power electromagnetic pulse source used for lithotripsy application. In such applications we have up to $80 \mathrm{MPa}$ in the focus, and we need a fine grid within the acoustic domain in order to resolve the higher harmonics forming the high-pressure pulse. The schematic setup of the electromagnetic pulse source is displayed in Fig. 17. When the slab coil is loaded by a capacitor discharge, eddy currents are induced in the aluminum plate. The interaction between these eddy currents and the overall magnetic field results in a magnetic volume force (Lorentz force) acting on the plate. Therewith, the aluminum plate is deformed and an acoustic pulse is radiated into the fluid and focused by the lens. Since in this case, we have to consider the non-linearities within the electromagnetic transducer, we perform the numerical simulation in two steps:

\section{(1) Transducer Computation}

Since the non-linearities of the acoustic field near the transducer can be neglected, we compute the acoustic pressure using the linear acoustic wave equation. Therewith, we fully take into account the fluid loading of the transducer. For modeling the electromagnetic transducer we consider all relevant non-linearities: (1) updated Lagrangian formulation for the magnetic field to take eddy currents due to the deformation of the aluminum plate within the magnetic field into account (motional electromagnetic force); (2) geometric non-linearity for the aluminum plate; (3) the non-linear electromagnetic 


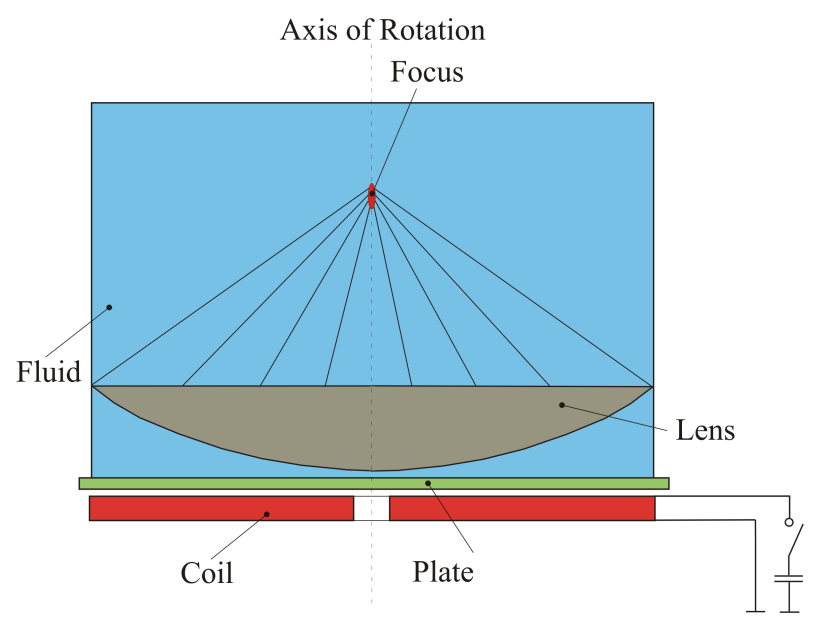

Fig. 17. Schematic of an electromagnetic driven acoustic power source

force term $[16,28]$.

\section{(2) Non-linear Wave Propagation Computation}

In a second run, we fully solve Kuznetsov's non-linear wave equation using the computed scalar acoustic potential near the transducer obtained from the first simulation step and prescribe it as Dirichlet boundary condition.

Therewith, in a first step we just consider the electromagnetic actuator and investigate the electromagnetic and mechanical quantities. This step has been performed for the full 3D model (due to the structure of the flat coil). The results are displayed in Fig. 18, where the eddy currents as well as the deformations of the aluminum plate are shown at different time steps. It can be clearly seen, that the deformations in radial direction are quite different. Especially at the center, the deformation is even in opposite direction. This results from the fact, that no eddy currents are induced in the aluminum plate near the center, since the flat coil has no turns there (see. Fig. 17). Therewith, in this region no Lorentz forces are generated, which could push this part of the aluminum plate away from the flat coil.

In a second step, we use the recorded scalar acoustic potential near the surface of the plate and prescribe it as Dirichlet boundary conditions within the FE formulation of Kuznetsov's equation for an axi-symmetric setup. The lense itself is modeled by the mechanical PDE, such that the fluid-structure interface between mechanics and acoustics is taken into account. For the numerical simulation a finite element grid width of $90 \mu \mathrm{m}$ (corresponds to about 70 linear finite elements per fundamental wavelength) was used for the acoustic domain, which resulted in already 2 million finite elements for the axisymmetric setup. The time step size has been chosen to include 100 time steps per fundamental time period.

The measured and simulated pressure signals in the focus region of the source are shown in Fig. 19. The dispersion at the beginning and the decreasing slope of the simulated pressure 


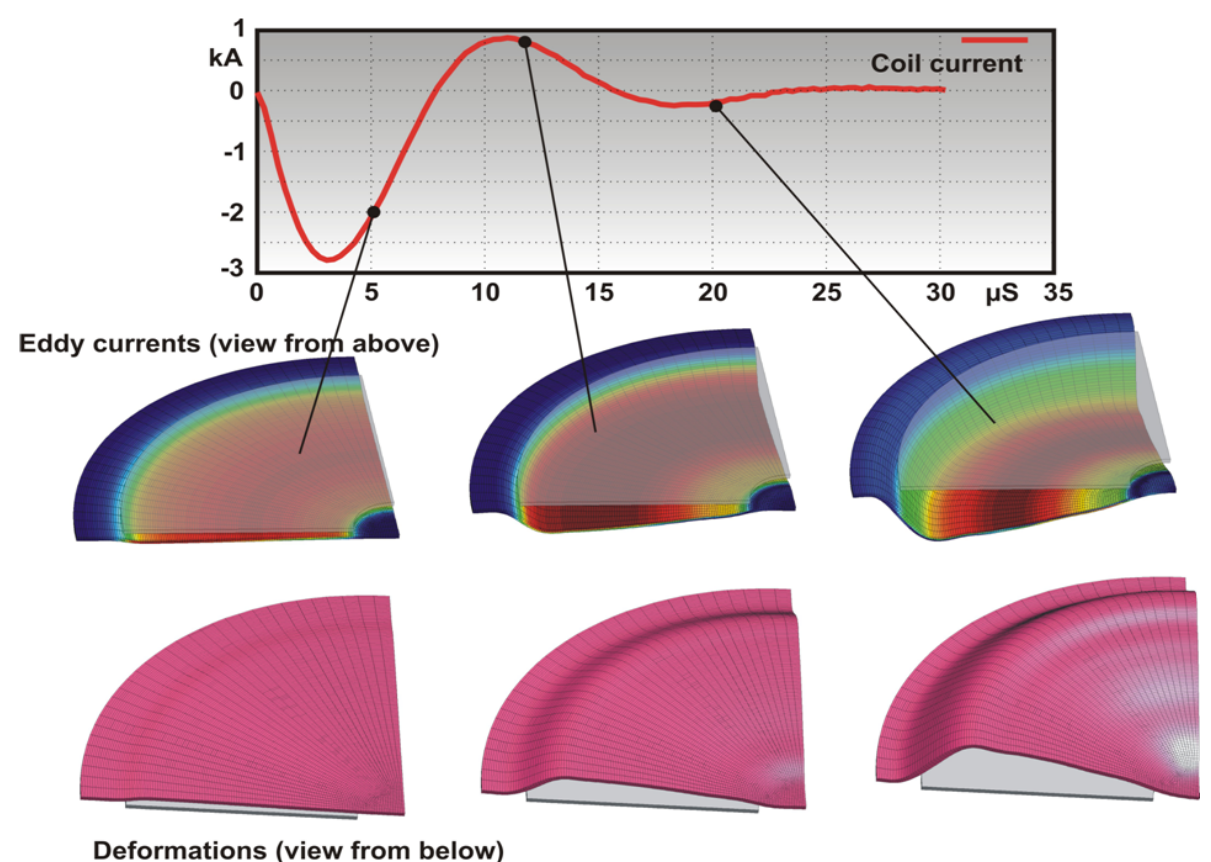

Fig. 18. Eddy currents and deformations of the aluminum plate at different time steps in relation to the resulting current in the flat coil.

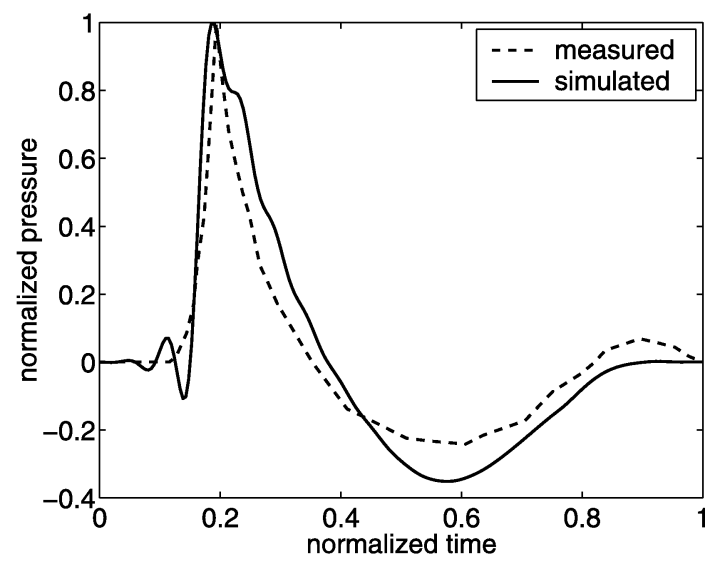

Fig. 19. Comparison between measured and simulated sound pressure level in the focal region of the electromagnetic pulse source

pulse as compared to the measured one indicates that the mesh size and the time step have to be reduced further to obtain higher accuracy. 


\section{Aero-acoustics}

Since the beginning of computational aero-acoustics several numerical methodologies have been proposed. Each of these trying to overcome the challenges that the specific problems pose for an effective and accurate computation of the radiated sound. The main difficulties include [29, 30]:

- Energy disparity and acoustic inefficiency: There is a large disparity between the overall energy of the flow and the part which is converted to acoustic energy. In general, the total radiated power of a turbulent jet scales with $O\left(v^{8} / c^{5}\right)$, and for a dipole source arising from pressure fluctuations on surfaces inside the flow scales with $O\left(v^{6} / c^{3}\right)$, where $v$ denotes the characteristic flow velocity and $c$ the speed of sound.

- Length scale disparity: A large disparity also occurs between the size of an eddy in the turbulent flow and the wavelength of the generated acoustic sound. Low Mach number eddies have a characteristic length scale $l$ and velocity $v$. This eddy will then radiate acoustic waves of the same characteristic frequency, but with a much larger length scale, expressed by the acoustic wavelength $\lambda$

$$
\lambda \propto c \frac{l}{v}=\frac{l}{M} .
$$

- Preservation of multipole character: The numerical analysis must preserve the multipole structure of the acoustic source. Furthermore, in order to estimate the source strength, it is necessary to resolve the whole structure of the source.

- Simulation of unbounded domains: As a main issue for the simulation of unbounded domains using volume discretization methods remains the boundary treatment which needs to be applied to avoid the reflection of the outgoing waves on the truncating boundary of the computational domain. This remains to be a very active field of research and several numerical formulations have been developed for both transient and harmonic analyses (for an overview we refer to $[6]$ ).

Currently available aeroacoustic methodologies overcome only some of these physical and numerical issues, which restricts their applicability and making them, in many cases, problem dependent methodologies. Due to the large disparities of length and energy scales between fluid and acoustic quantities, Direct Numerical Simulation (DNS) still remains restricted to low Reynolds number flows [31,32]. Due to the practical advantages provided by the separate treatment of fluid and acoustic computations, these hybrid methodologies still remain the most commonly used approaches for aeroacoustic computations. Among the group of hybrid approaches, integral methods remain widely used in CAA for solving large open acoustic domain problems such as airframe noise, landing gear noise, fan (turbines) noise and rotor noise. In such cases, integral methods such as Lighthill's acoustic analogy (see, e.g., [33,34]), Curle's formulation (see, e.g., [35]), Ffwocs-Williams and Hawkings' formulation (see, e.g., [35-37]), the Kirchhoff method (see, e.g., $[37,38]$ ) or extensions thereof 
(see, e.g., [39-42]) are computationally less expensive than volume discretization methods where a volume discretization of the acoustic domain is required. In case of elastic boundaries or for the inclusion of structural mechanics - acoustics effects, volume discretization methods are necessary to account directly for the interaction between solid and fluid. In these cases, integral formulations would require a priori knowledge of a hard-wall Green's function that is not known for complex geometries [43]. Among the volume discretization methods used in CAA we find finite differences (FD), discontinuous Galerkin (DG) and finite volume (FV) schemes, generally employed to solve aeroacoustic formulations based on Linearized Euler Equations (see, e.g., [44-49]) or systems of equations such as the Acoustic Perturbation Equation (see [50]) and Linearized Perturbed Compressible Equation (see [51]). Additionally, we find the Finite Element (FE) method used to solve the variational formulation of Lighthill's acoustic analogy $[52,53]$.

Figure 20 shows the typical numerical methods which are employed when using one of these hybrid methodologies. For a precise prediction of the flow induced noise, Large Eddy

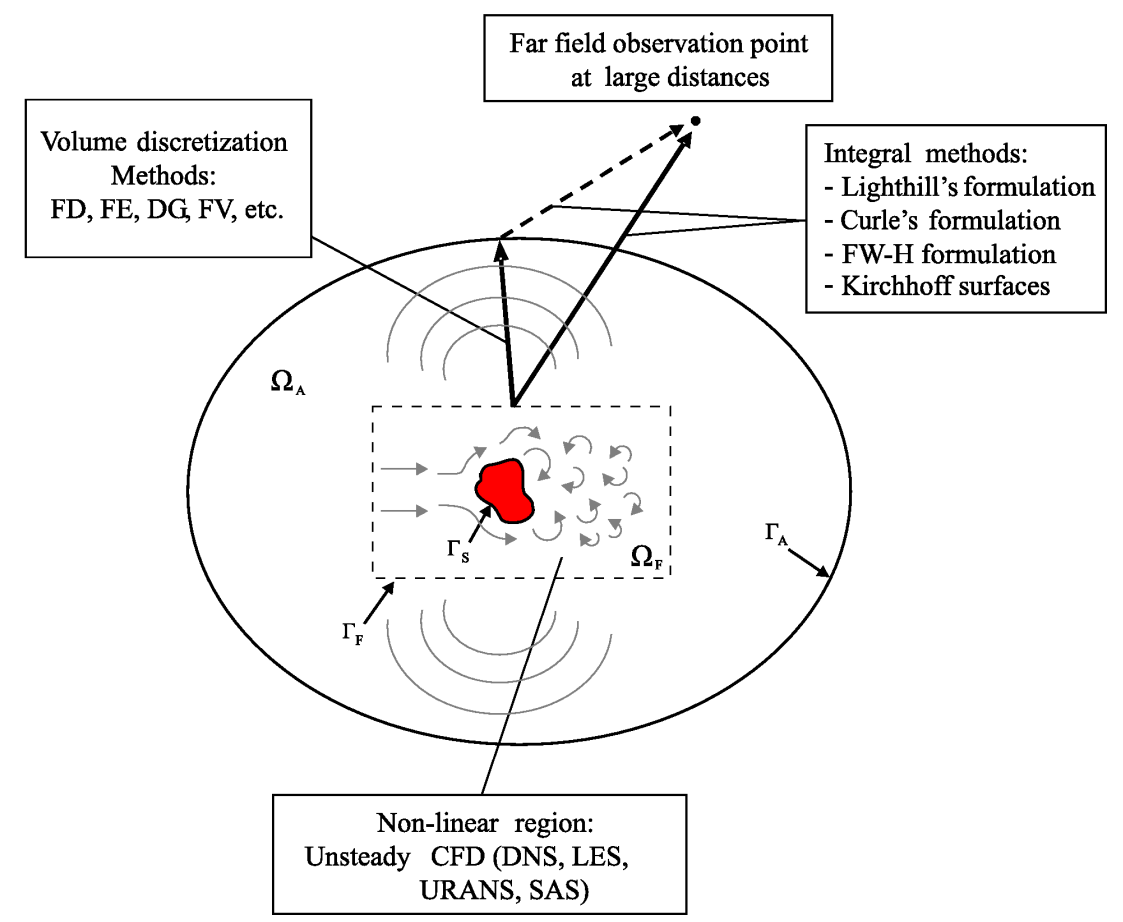

Fig. 20. Schematic of some of the possible strategies when using an aeroacoustic hybrid approach.

Simulation (LES) is mainly used for solving the flow and provide the acoustic sources. In the LES method the large scales are directly resolved and the effect of the small scales on the large scales are modeled [54]. Even though the computational cost is still high, it is possible to simulate turbulent flows with high Reynold numbers and complex geometries with the LES method. Therefore, this method has been adopted by researchers as one of the 
standard methods for computing the turbulent near field in CAA problems. Additionally we find the group of combined RANS (Reynolds Averaged Navier Stokes)/LES methods [55]. This type of methods blend statistical approaches with LES in order to yield enhanced predictions of both turbulence statistics and unsteady flow dynamics at a fraction of the cost of traditional LES. Under these RANS/LES methods we find Detached Eddy Simulation (DES) [56] which allows the turbulence model to pass from an uRANS (unsteady Reynolds Averaged Navier Stokes) method for attached boundary layers to a LES in separated regions. Another recently developed approach is the Scale-Adaptive Simulation (SAS) method [57], which adjusts to the already resolved scales in a dynamic way and allows the development of a turbulent spectrum in the detached regions. Therefore, it shows similar characteristics as a DES model, but without the explicit grid dependence in the RANS regime [57]. Furthermore, to keep the computational costs for the CFD computation as low as possible, there are many attempts to use RANS and to generate a time dependent velocity field by help of the SNGR (Stochastic Noise Generation and Radiation) method, which is used to evaluate acoustic source terms (see, e.g., [58-60]). These approaches are strongly used to predict broadband noise, e.g., [61,62]. A review of the current ongoing research in CFD methods used for CAA simulations can be found in [63].

Many hybrid approaches employed in CAA are based on the inhomogeneous wave equation as derived by Lighthill in [34], which reads as follows

$$
\frac{1}{c^{2}} \frac{\partial^{2} p^{\prime}}{\partial t^{2}}-\frac{\partial^{2} p^{\prime}}{\partial x_{i}^{2}}=\frac{\partial^{2} L_{i j}}{\partial x_{i} \partial x_{j}}
$$

In (32) $L_{i j}$ denotes Lighthill's stress tensor given by

$$
L_{i j}=\rho v_{i} v_{j}+\delta_{i j}\left[\left(p-p_{0}\right)-c_{0}^{2}\left(\rho-\rho_{0}\right)\right]-e_{i j}
$$

with $v_{i}$ the $i$-th component of the flow velocity vector $\mathbf{v}, p$ the overall pressure, $e_{i j}$ the $(i, j)$ th component of the viscous stress tensor, $\rho_{0}$ and $p_{0}$ the atmospheric values of density and pressure, respectively.

An important aspect in Lighthill's acoustic analogy is the assumption that $L_{i j}$ is known or can be evaluated to a certain degree of approximation. Additionally, this source term is assumed to vanish outside the turbulent region. For a turbulent flow embedded in a uniform atmosphere at rest, Lighthill's stress tensor $L_{i j}$ can be neglected outside the turbulent region itself. Moreover, the effects of viscosity and heat conduction are expected to cause only a slow damping due to the conversion of acoustic energy into heat and to have a significant effect only for very large distances. Under these assumptions, it is possible to neglect $e_{i j}$ entirely [33].

Furthermore, the term $\left(p-p_{0}\right)-c_{0}^{2}\left(\rho-\rho_{0}\right)$ only becomes important for anisotropic media, when the Mach number in the acoustic domain is significantly different from the one in the fluid domain. For isentropic flows in which $\left(p-p_{0}\right) / p_{0}$ and $\left(\rho-\rho_{0}\right) / \rho_{0}$ are very small, the relation

$$
\left(p-p_{0}\right)=c_{0}^{2}\left(\rho-\rho_{0}\right)
$$


can be assumed. Therefore, the resulting approximation of Lighthill's tensor is given by

$$
L_{i j} \approx \rho v_{i} v_{j}
$$

The explicit separation of propagation and generation as derived by Lighthill has raised many discussions ever since, which motivated the derivation of improved acoustic analogy formulations. A difficulty of Lighthill's equation is the interpretation of the source term where mean flow effects on the wave propagation are included [64]. In order to obtain a formulation to describe the noise propagation in a transversally sheared mean flow, Lilley $[65,66]$ proposed a third-order wave operator. The mean flow and any refraction it causes are explicitely considered in this wave operator.

\subsection{FE Formulation for Aero-acoustics}

Let us consider the global acoustic domain subdivided into two sub-domains for the source and far field regions (cf. Fig. 21). Here $\Omega_{\mathrm{a} 1}$ denotes the acoustic source region (corresponding to the computational domain for the fluid flow), and $\Omega_{\mathrm{a} 2}$ the far field propagation domain. Furthermore, we consider in $\Omega_{\mathrm{a} 1}$ a solid domain $\Omega_{\mathrm{s}}$ with a flow - structural mechanics interface along $\Gamma_{\mathrm{fs}}$. Therewith, we solve (32) in $\Omega_{\mathrm{a} 1}$ and (5) in $\Omega_{\mathrm{a} 2}$ to obtain the acoustic pressure $p^{\prime}: \Omega_{i} \times(0, T) \rightarrow \mathbb{R}$. In order to accurately resolve the aeroacoustic source term,

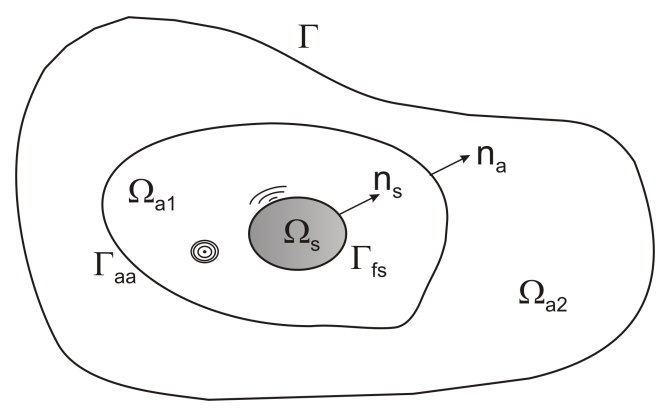

Fig. 21. Geometrical setup for aero-acoustics.

our approach is to employ a much finer spatial discretization in $\Omega_{\mathrm{a} 1}$ than in $\Omega_{\mathrm{a} 2}$ without the requirement of geometrical conformity between them. Therewith, we obtain a nonmatching interface $\Gamma_{\text {aa }}$, where adequate coupling conditions have to be applied. We cannot guarantee pointwise continuity of the acoustic pressure as would be the case for geometrically conforming triangulations,

$$
p_{1}^{\prime}-p_{2}^{\prime}=0 \text { on } \Gamma_{\text {aa. }}
$$

Therefore, we reformulate this condition as a weak condition for the jump of the pressure on both sides of the interface and choose $\mu$ from a suitable Lagrange multiplier space $M$ 
(cf. [9])

$$
\int_{\Gamma_{\mathrm{aa}}}\left(p_{1}-p_{2}\right) \mu \mathrm{d} \Gamma=0 \quad \forall \mu \in M .
$$

We also postulate that the flux of the acoustic pressure is continuous across $\Gamma_{\mathrm{aa}}$. We do this by introducing a Lagrange multiplier (LM)

$$
\lambda=-\frac{\partial p_{1}^{\prime}}{\partial \mathbf{n}_{\mathrm{a}}}=-\frac{\partial p_{2}^{\prime}}{\partial \mathbf{n}_{\mathrm{a}}} \text { on } \Gamma_{\mathrm{aa}} .
$$

The sub-domain from which the discretization for the Lagrange multiplier on the interface is inherited is called slave side. The values of the functions defined on it are determined by the values of the functions on the other side, which is called master side. We use the finite element basis functions restricted to the discretization of the interface on the slave side as Lagrange multiplier (cf. [9]).

We rewrite the weak formulation of the acoustic wave equations (see (32) and (5)) for the two sub-domains and substitute the definition of the Lagrange multiplier according to (36). For simplicity, we set the arising integral over the outer boundary $\Gamma$ to zero . Summing up, one arrives at the symmetric saddle point problem of finding $p_{1}^{\prime}, p_{2}^{\prime}, \lambda$ such that

$$
\begin{aligned}
& \sum_{i=1}^{2}\left(\int_{\Omega_{\mathrm{a} i}} \frac{1}{c^{2}} w_{i} \ddot{p}_{i}^{\prime} \mathrm{d} \Omega+\int_{\Omega_{\mathrm{a} i}} \nabla w_{i} \cdot \nabla p_{i}^{\prime} \mathrm{d} \Omega\right)+\int_{\Gamma_{\mathrm{aa}}}\left(w_{1}-w_{2}\right) \lambda \mathrm{d} \Gamma \\
& \quad+\int_{\Gamma_{\mathrm{fs}}} w_{1} \frac{\partial p^{\prime}}{\partial \mathbf{n}_{\mathrm{s}}} \mathrm{d} \Gamma=-\int_{\Omega_{\mathrm{a} 1}}\left(\nabla \cdot L_{i j}\right) \cdot \nabla w_{1} d \Omega+\int_{\Gamma_{\mathrm{fs}}} w_{1}\left(\nabla \cdot L_{i j}\right) \cdot \mathbf{n}_{\mathrm{s}} \mathrm{d} \Gamma, \\
& \int_{\Gamma_{\mathrm{aa}}}\left(p^{\prime}{ }_{1}-p^{\prime}{ }_{2}\right) \mu \mathrm{d} \Gamma=0
\end{aligned}
$$

for all $\mu$ and $w_{i}, i=1,2$. We note that $\mu$ is a test function with the same basis as the LM $\lambda$. The boundary integral over $\Gamma_{\mathrm{fs}}$ including the Lighthill tensor is obtained by applying integration by parts. It may be substituted based on the conservation of flow momentum by $[53]$

$$
\int_{\Gamma_{\mathrm{fs}}} w_{1}\left(\nabla \cdot L_{i j}\right) \cdot \mathbf{n}_{\mathrm{s}} \mathrm{d} \Gamma=\int_{\Gamma_{\mathrm{fs}}} w_{1} \frac{\partial p^{\prime}}{\partial \mathbf{n}_{\mathrm{s}}} \mathrm{d} \Gamma-\int_{\Gamma_{\mathrm{fs}}} w_{1} \frac{\partial \rho \mathbf{v}}{\partial t} \cdot \mathbf{n}_{\mathrm{s}} n_{i} \mathrm{~d} \Gamma .
$$

The term $\int_{\Gamma_{\mathrm{fs}}} w_{1} \partial p^{\prime} / \mathbf{n}_{\mathrm{s}} \mathrm{d} \Gamma$ in (39) cancels, when being substituted into (37). According to the continuity between the flow velocity and the mechanical velocity of the solid along the common interface $\Gamma_{\mathrm{fs}}$, we may substitute the flow velocity $\mathbf{v}$ by the solid velocity $\mathbf{v}_{\mathrm{s}}$. Furthermore, the vibrating solid body will generate acoustic sound according to the coupling condition (4). Therefore, the extended weak formulation of Lighthill's wave equation considering both flow induced and vibrational induced sound with a nonconforming interface 
between the source and propagation regions reads as

$$
\begin{aligned}
\sum_{i=1}^{2}\left(\int_{\Omega_{\mathrm{a} i}} \frac{1}{c^{2}} w_{i} \ddot{p}_{i}^{\prime} \mathrm{d} \Omega+\int_{\Omega_{\mathrm{a} i}} \nabla w_{i} \cdot \nabla p^{\prime}{ }_{i} \mathrm{~d} \Omega\right) & +\int_{\Gamma_{\mathrm{aa}}}\left(w_{1}-w_{2}\right) \lambda \mathrm{d} \Gamma \\
& =-\int_{\Omega_{\mathrm{a} 1}}\left(\nabla \cdot L_{i j}\right) \cdot \nabla w_{1} d \Omega+\int_{\Gamma_{\mathrm{fs}}} \rho_{0} w_{1} \frac{\partial^{2} \mathbf{u}}{\partial t^{2}} \cdot \mathbf{n}_{\mathrm{s}} \mathrm{d} \Gamma, \\
\int_{\Gamma_{\mathrm{aa}}}\left(p^{\prime}{ }_{1}-p^{\prime}{ }_{2}\right) \mu \mathrm{d} \Gamma & =0 .
\end{aligned}
$$

Performing a spatial discretization using Lagrangian finite elements, we arrive at the following semi-discrete Galerkin formulation

$$
\left(\begin{array}{ccc}
\mathbf{M}_{p 1} & 0 & 0 \\
0 & \mathbf{M}_{p 2} & 0 \\
0 & 0 & 0
\end{array}\right)\left(\begin{array}{l}
\ddot{p}_{1} \\
\underline{\ddot{p}_{2}} \\
\underline{\ddot{\lambda}}
\end{array}\right)+\left(\begin{array}{ccc}
\mathbf{K}_{p 1} & 0 & \mathbf{D} \\
0 & \mathbf{K}_{p 2} & \mathbf{M} \\
\mathbf{D}^{T} & \mathbf{M}^{T} & 0
\end{array}\right)\left(\begin{array}{l}
\underline{p_{1}} \\
\underline{p_{2}} \\
\underline{\lambda}
\end{array}\right)=\left(\begin{array}{l}
\underline{f_{1}} \\
\underline{0} \\
\underline{0}
\end{array}\right) .
$$

In (42) $\mathbf{M}_{p 1}, \mathbf{M}_{p 2}$ and $\mathbf{K}_{p 1}, \mathbf{K}_{p 2}$ are the mass and stiffness matrices respectively. The matrices $\mathbf{D}$ and $\mathbf{M}$ are due to the nonconforming interface and are formally mass matrices. They compute element-wise as

$$
\begin{aligned}
& \mathbf{D}=\bigwedge_{e=1}^{n_{\mathrm{es}}} \mathbf{d}^{e} ; \quad \mathbf{d}^{e}=\left[d_{a b}\right] ; \quad d_{a b}=\int_{\Gamma_{e}} N_{a}^{j} \Phi_{b}^{j} \mathrm{~d} \Gamma, \\
& \mathbf{M}=\bigwedge_{e=1}^{n_{\text {isec }}} \mathbf{m}^{e} ; \quad \mathbf{m}^{e}=\left[m_{a b}\right] ; \quad m_{a b}=\int_{\Gamma_{e}} N_{a}^{k} \Phi_{b}^{j} \mathrm{~d} \Gamma .
\end{aligned}
$$

For all details, concerning the intersection operators and the evaluation of the above matrices, we refer to [10]. This advanced formulation is within the framework of Mortar FEM, see, e.g., $[9,67,68]$.

A crucial point for any hybrid method is the transfer of the acoustic sources from the flow grid to the acoustic grid $[53,69]$. In order to preserve the acoustic energy, we compute the source terms within the FE formulation on the fine flow grid, which we then interpolate to the coarser acoustic grid (see Fig. 22(a)). This interpolation has to be conservative to ensure the preservation of acoustic energy. Therefore, for each of these nodal sources $f_{k}^{\mathrm{F}}$ on the flow grid we have to find its position in the acoustic grid. We then compute from the global position $\left(x_{k}, y_{k}\right)$ the local position $\left(\xi_{k}, \eta_{k}\right)$ in the reference element (see Fig. 22(b)), which is in general a non-linear mapping and is solved by a Newton scheme. Now, it is possible to perform a bilinear interpolation and add the contribution of $f_{k}^{\mathrm{F}}$ to the nodes of the acoustic grid by using standard finite element basis functions $N_{i}$ corresponding

$$
f_{i}^{\mathrm{A}}=N_{i}\left(\xi_{k}, \eta_{k}\right) f_{k}^{\mathrm{F}} .
$$

By this procedure, the interpolation preserves the overall energy of the acoustic sources. 


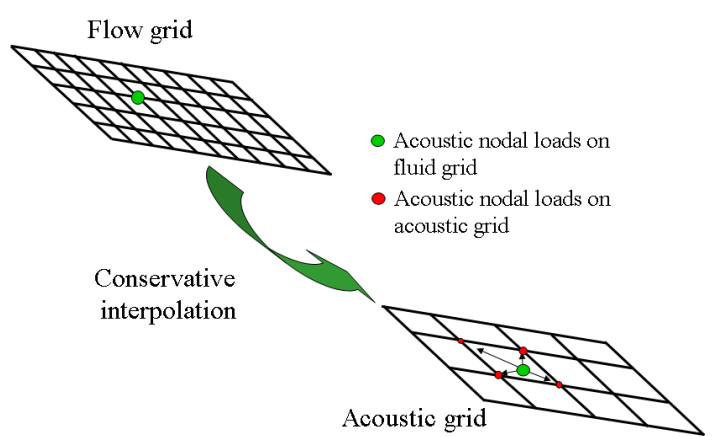

(a) Illustration of conservative interpolation.

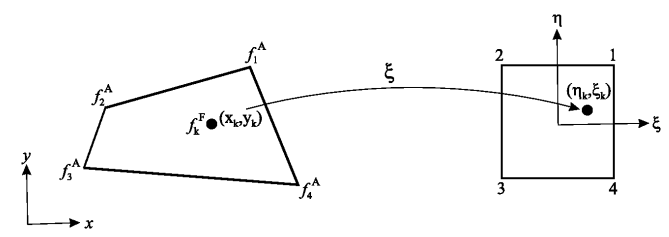

(b) Global to local mapping.

Fig. 22. Conservative interpolation.

\subsection{Application: Human Phonation}

Besides respiration itself, the human respiratory system has a second important functionality: phonation. The possibility to generate sounds is an important factor in social life as therewith information can be exchanged. The larynx plays an important role in this process. It is located inside the throat and connects either the trachea (airway) with the pharynx or the esophagus (food pipe or digestive tract). The larynx can be understood as a valve that has two positions, one for breathing, where the trachea is connected to the pharynx and one for swallowing, where the esophagus is connected to the pharynx. During exhalation the larynx can generate sounds by setting the vocal folds into the phonation position. Figure 23 shows the frontal cut through the larynx. While in Fig. 23(a) the anatomical components

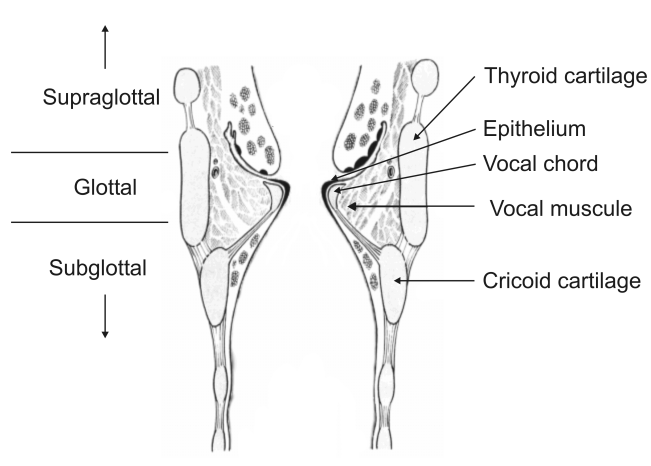

(a) rontal cut through the larynx.

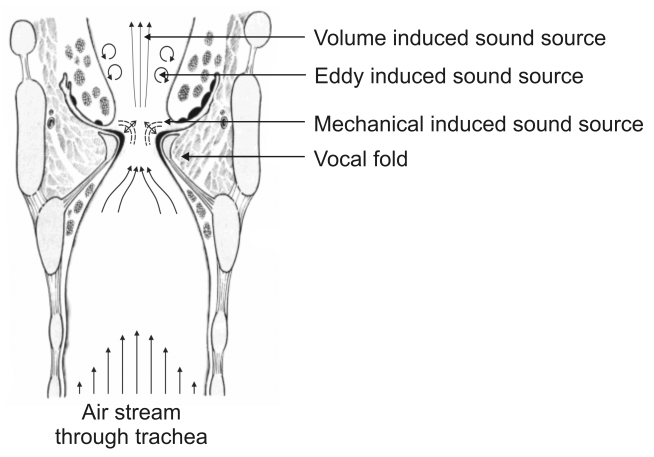

(b) Different sound mechanism.

Fig. 23. Frontal cut through the larynx showing the anatomical constitution and the different sound sources.

are specified, Fig. 23(b) illustrates the sound sources that exist in phonation. Basically, we differ between three sources: the eddy-induced sound which emerges from turbulent flow 
structures, the volume-induced sound due to the modulated flow resulting from the selfsustained oscillation of the vocal folds, and the sound from the vibrating vocal folds. The modulated flow provides the main contribution. The air stream induced by lung compression is guided through the trachea and through the glottis. The air stream induces the vocal folds to vibrate yielding an oscillating glottis cross sectional area. Due to the varying cross sectional area the air stream gets modulated in an acoustically relevant frequency range. This process builds the fundamental frequency of phonation.

For a realistic physical model of the phonation process, we have to consider the multifield setup of fluid, solid-mechanics and acoustics. The fluid flow through the larynx is modeled with the incompressible Navier-Stokes equations

$$
\begin{aligned}
\rho \frac{\partial \mathbf{v}}{\partial t}+\rho(\mathbf{v} \cdot \nabla) \mathbf{v}+\nabla p-\mu \Delta \mathbf{v} & =0 \\
\nabla \cdot \mathbf{v} & =0
\end{aligned}
$$

with $\mathbf{v}$ the flow velocity, $\rho$ the fluid density, $p$ the hydrodynamic pressure and $\mu$ the dynamic viscosity. The equations hold for incompressible fluids, which may be assumed due to the fact that for the considered application the Mach number is smaller than 0.3. The computational domain of the fluid flow constantly changes since the vocal folds move and hence define the fluid boundary. This difficulty is tackled by utilizing the Arbitrary-Lagrangian-Eulerian (ALE) approach (for details see [70,71]).

The air and vocal folds share a common interface $\Gamma_{\mathrm{fs}}$ along the continuity between the fluid velocity $\mathbf{v}$ and the mechanical velocity $\mathbf{v}_{\mathrm{s}}$ (expressed by the first time derivative of the solid displacement $\mathbf{u}$ ) has to hold

$$
\mathbf{v}=\frac{\partial \mathbf{u}}{\partial t} \quad \text { on } \Gamma_{\mathrm{fs}} .
$$

This implies for solid mechanics the following inhomogeneous Neumann boundary condition

$$
\left[\boldsymbol{\sigma}_{\mathrm{s}}\right] \cdot \mathbf{n}=\left[\boldsymbol{\sigma}_{\mathrm{f}}\right] \cdot \mathbf{n} \quad \text { on } \Gamma_{\mathrm{fs}}
$$

describing the equivalence of fluid stress $\left[\boldsymbol{\sigma}_{\mathrm{f}}\right]$ and solid stress $\left[\boldsymbol{\sigma}_{\mathrm{s}}\right]$ in normal direction $\mathbf{n}$. The fluid stresses can be written explicitly by the hydrodynamic pressure $p$ and fluid velocity $\mathbf{v}$ as

$$
\left[\boldsymbol{\sigma}_{\mathrm{s}}\right] \cdot \mathbf{n}=\rho_{0} \underbrace{\int_{\Gamma_{\text {fs }}}-p \mathbf{I} \cdot \mathbf{n} \mathrm{d} \Gamma}_{\text {pressure }}+\underbrace{\int_{\Gamma_{\text {fs }}} \mu\left(\nabla \mathbf{v}+(\nabla \mathbf{v})^{t} \cdot \mathbf{n}\right) \mathrm{d} \Gamma}_{\text {shear }} .
$$

Having Dirichlet boundary condition for the fluid and Neumann boundary conditions for solid mechanics, the fluid-solid interaction is also called Dirichlet-to-Neumann problem.

A sketch of all relevant relationships between the physical fields is given in Fig. 24, which has been implemented using the finite element (FE) method [72,73]. Generally speaking, we consider a fluid flow which acts onto a deformable structure, which in turn influences 


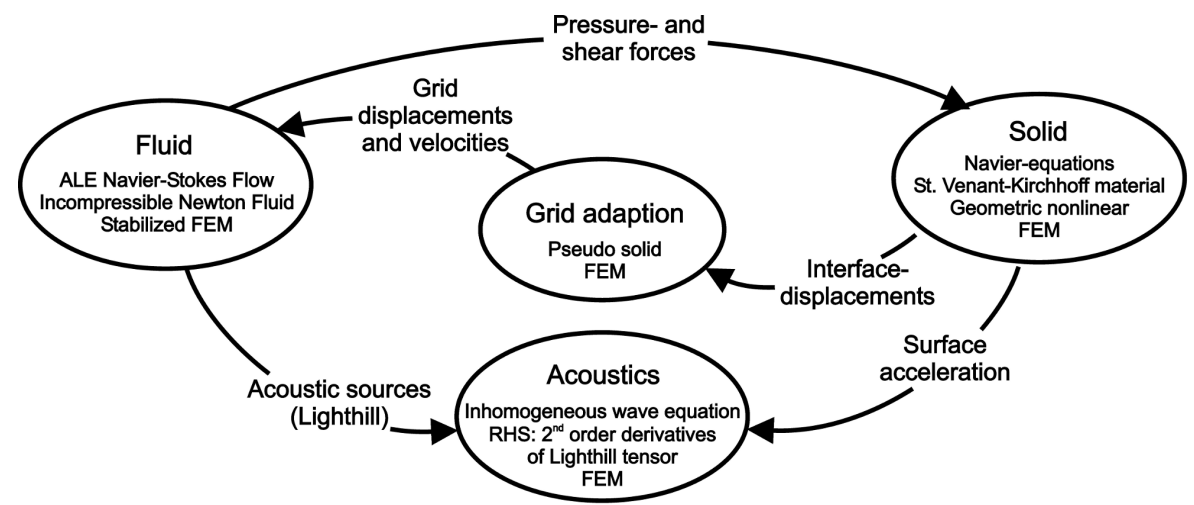

Fig. 24. Sketch of interaction and coupling types between fluid mechanics, structure mechanics and acoustics.

the adhering fluid. Furthermore, the deforming structure prescribes the fluid domain and its grid which has to change constantly in time (ALE method).

In the following we will present simulation results for a simplified geometrical model of the larynx. This simulation setup consists of a channel with the two vocal folds, which act as a constriction inside the channel as displayed in Fig. 25(a). Figure 25(b) shows the fine mesh around the vocal folds, which is necessary to accurately resolve the fluid flow. Approximately 45000 quadratic finite elements are used to resolve the fluid, which results in about 400000 degrees of freedom. For structural mechanics the vocal folds have been divided into three different layers, the body, the ligament and the cover. Each have different elasticity modulus to model the real physiology more accurately. For body, ligament and cover the elasticity moduli were set to $21 \mathrm{kPa}, 33 \mathrm{kPa}$ and $12 \mathrm{kPa}$ respectively. To simulate the pressure, the lungs build up, a pressure gradient from in- to outflow of $1.5 \mathrm{kPa}$ is prescribed. The simulations show the typical self sustained oscillation of the vocal folds

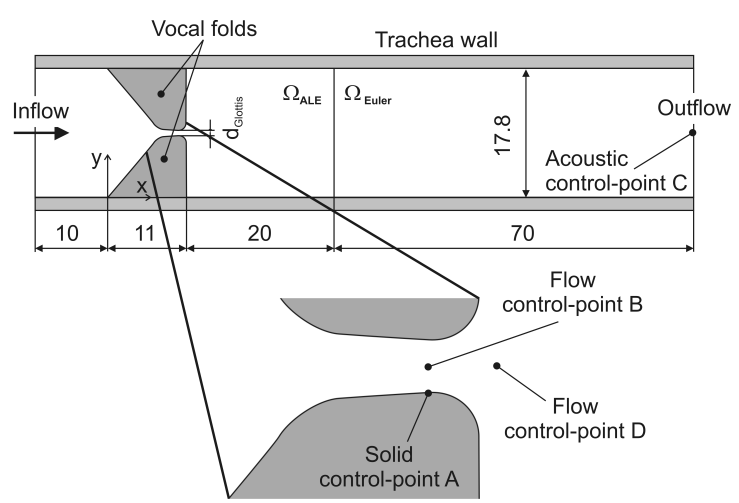

(a) Simulation model (dimensions in) $\mathrm{mm}$.

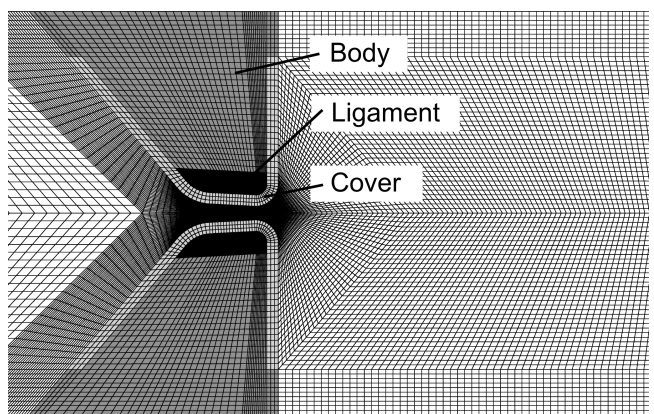

(b) Mesh around the vocal folds.

Fig. 25. Model of the larynx with vocal folds and the according mesh used for the simulations. 
during phonation, which is divided into the divergent (opening) and convergent (closing) phase as displayed in Fig. 26. In Fig. 27 the fluid field can be seen at a characteristic time
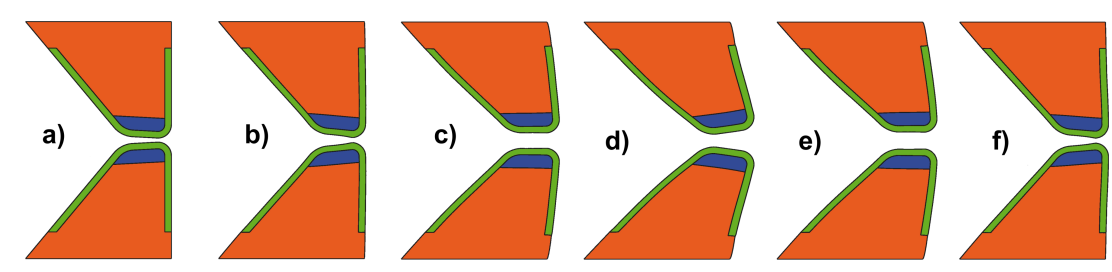

Fig. 26. Computed deformation cycle of the vocal folds, which can be divided in divergent to convergent phase.

step. In the transient simulation one can observe, how the jet is stochastically attaching to either side of the trachea wall, which is known as the Coanda effect.

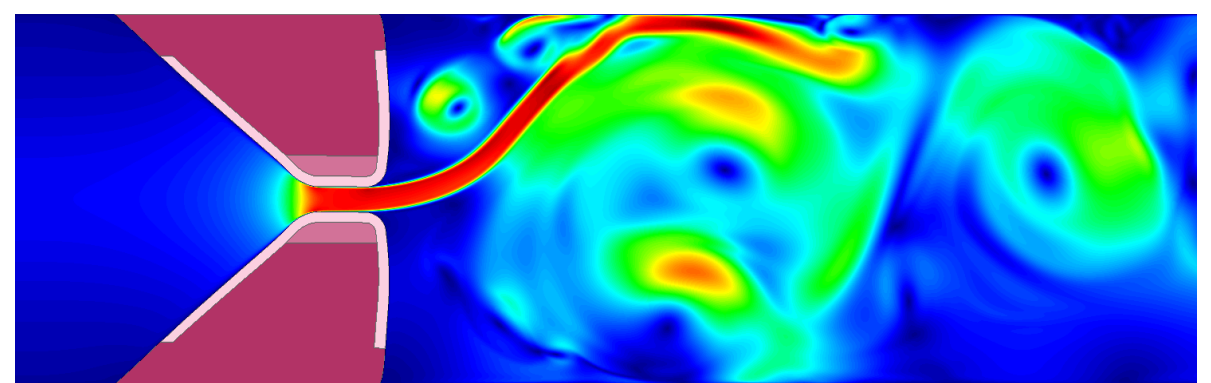

Fig. 27. Snap-shot of velocity field and deformation of vocal folds. Jet is attached to the top vocal fold Coanda effect.

The acoustic sound computation has been separated into a computation of the flow induced sound (using Lighthill's tensor, see (35)) and into a vibrational induced sound (see (4) for the coupling term ). In a series of simulations the acoustic field of vibrational and fluid induced sound was compared. As can be seen in Fig. 28(a) the mechanical induced sound is much smaller than that of the fluid induced sound. Comparing this result with a simulation were the initial glottis width is enlarged to $0.7 \mathrm{~mm}$ (see Fig. 28(b)) it shows that the bigger glottis results in a much broader acoustic frequency spectrum. Furthermore, no dominant frequency component is recognizable as in Fig. 28(a) at about $190 \mathrm{~Hz}$.

These results imply the importance of a proper closing glottis for a clear and healthy voice. Furthermore, they show that the fluid flow is the dominant source of the phonation, which is hard to proof by measurements.

\section{Conclusion}

We have presented physical/mathematical models for computational acoustics based on partial differential equations and their efficient numerical solution applying the FE method. 


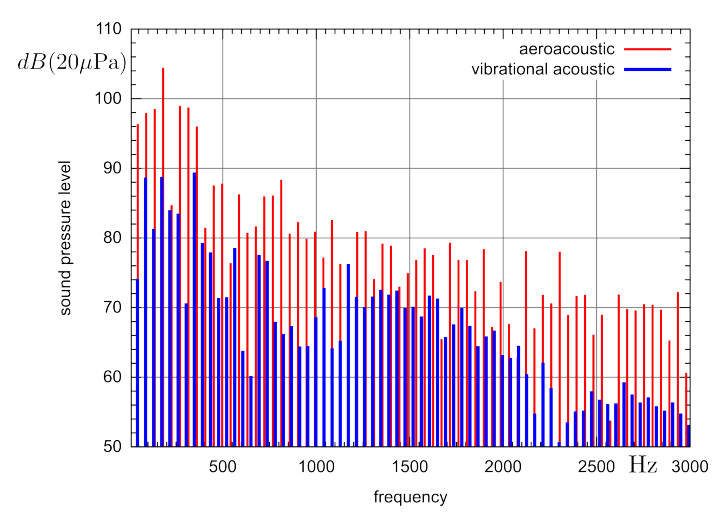

(a) Acoustic spectra of vibrational and fluid induced sound at a glottis width of $0.3 \mathrm{~mm}$

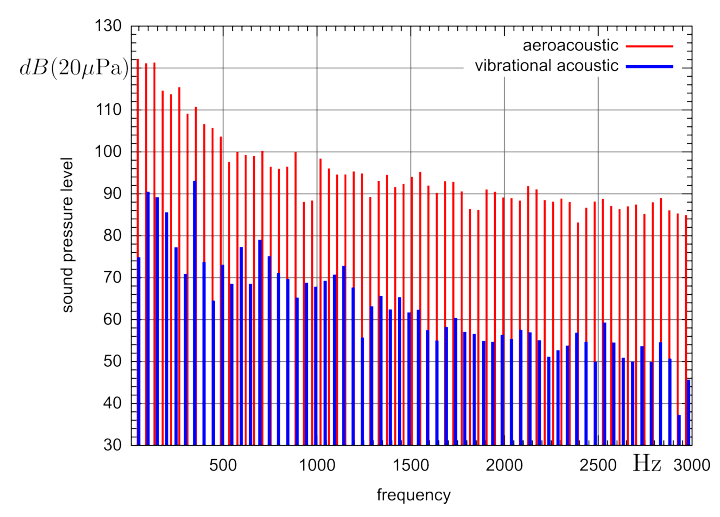

(b) Acoustic spectra of vibrational and fluid induced sound at a glottis width of $0.7 \mathrm{~mm}$.

Fig. 28. Comparison of acoustic spectra for fluid induced and vibrational induced sound simulation for different glottis widths.

Therewith, we have concentrated on three topics: vibro-acoustics, aero-acoustics and highintensity focused ultrasound. All these topics have in common, that they are multi-field problems, where the acoustic field is the field of main interest. We are aware of the fact, that our presentation is just a small extract of the broad interdisciplinary field acoustics has developed. However, the application examples demonstrates the importance of numerical simulation schemes being able to handle multi-field problems.

\section{Acknowledgment}

The author wants to gratefully acknowledge his Ph.D. students Simon Triebenbacher, Stefan Zörner, Andreas Hüppe and Andreas Hauck as well as the cooperation with Barbara Wohlmuth and Igor Shevchenkoa on Mortar FEM. Furthermore, I gratefully thank the editor of this special issue for his valuable comments and proof reading.

This work is funded through the Austrian Science Foundation (FWF) grant I 533-N20 and German Science Foundation (DFG) grant WO 671/6-1.

\section{References}

1. T. D. Rossing, ed., Handbook of Acoustics. Springer, 2007.

2. M. Kaltenbacher, "Advanced Simulation Tool for the Design of Sensors and Actuators," in Proceedings of Eurosensors XXIV, (Linz, Austria), September 5-8 2010. CD-ROM.

3. M. Kaltenbacher and R. Lerch, "Perfectly Matched Layer Technique for the Numerical Computation of Wave Propagation Phenomena," in Proceedings of the 2007 IEEE International Ultrasonics Symposium, pp. 183-186, 2007. New York, 28.-31.10.2007.

4. M. Rausch, M. Kaltenbacher, H. Landes, R. Lerch, J. Anger, J. Gerth, and P. Boss, "Combination of Finite and Boundary Element Methods in Investigation and Prediction of Load- 
Controlled Noise of Power Transformers," Journal of Sound and Vibration, vol. 250, no. 2, pp. 323-338, 2002.

5. M. Kaltenbacher, Numerical Simulation of Mechatronic Sensors and Actuators. Berlin: Springer, 2. ed., 2007. ISBN: 978-3-540-71359-3.

6. D. Givoli, "Computational absorbing boundaries," in Computational Acoustics of Noise Propagation in Fluids (S. Marburg and B. Nolte, eds.), ch. 5, pp. 145-166, Berlin Heidelberg: SpringerVerlag, 2008.

7. B. Engquist and A. Majda, "Absorbing Boundary Conditions for the Numerical Simulation of Waves," Mathematics of Computation, vol. 31, pp. 629-651, 1977.

8. R. Adams, Sobolev Spaces. Pure and Applied Mathematics, Academic Press, 1975.

9. B. Flemisch, M. Kaltenbacher, and B. Wohlmuth, "Elasto-Acoustic and Acoustic-Acoustic Coupling on Nonmatching Grids," Int. J. Numer. Meth. Engng, vol. 67, no. 13, pp. 1791-1810, 2006.

10. S. Triebenbacher, M. Kaltenbacher, B. Flemisch, and B. Wohlmuth, "Applications of the mortar fem in elasto-acoustics and flow induced noise computations," Acta Acustica, vol. 96, pp. 536553,2010

11. T. J. R. Hughes, The Finite Element method. New Jersey: Prentice-Hall, 2000.

12. F. B. Belgacem, "The mortar finite element method with lagrange multipliers," Numer. Math., vol. 84, no. 2, pp. 173-197, 1999.

13. B. Wohlmuth, "A mortar finite element method using dual spaces for the lagrange multiplier," SIAM J. Numer. Anal., vol. 38, no. 3, pp. 989-1012, 2000.

14. D. Braess and M. Kaltenbacher, "Efficient 3d-finite-element-formulation for thin mechanical and piezoelectric structures," International Journal for Numerical Methods in Engineering, vol. 73, no. 2, pp. 147-161, 2008.

15. J. Berenger, "A perfectly matched layer for the absorption of electromagnetic waves," J. Comp. Phys., vol. 114, 1994.

16. M. Kaltenbacher, H. Landes, J. Hoffelner, and R. Simkovics, "Use of Modern Simulation for Industrial Applications of High Power Ultrasonics," in Proceedings of the IEEE Ultrasonics Symposium, CD-ROM Proceedings, pp. 673-678, IEEE, 2002. München, 08.-11.10.2002.

17. V. Kuznetsov, "Equations of Nonlinear Acoustics," Soviet Physics - Acoustics, vol. 16, no. 4, pp. 467-470, 1971.

18. M. Hamilton and D. Blackstock, Nonlinear Acoustics. Academic Press, 1998.

19. H. Pennes, "Analysis of tissue and arterial blood temperature in the resting human forearm," The Journal of Applied Physiology, vol. 1, no. 2, pp. 93-122, 1948.

20. W. Nyborg, "Heat generation by ultrasound in a relaxing medium," J. Acoust. Soc. Am., vol. 70, no. 2, pp. 310-312, 1981.

21. T. Cavicchi and W. J. O'Brien, "Heat generation by ultrasound in an absorbing medium," $J$. Acoust. Soc. Am., vol. 76, no. 4, pp. 1244-1245, 1984.

22. D. Blackstock, "Connection between the Fay and Fubini Solutions for Plane Sound Waves of Finite Amplitude," J. Acoust. Soc. Am., vol. 39, no. 6, pp. 1019-1026, 1966.

23. M. Kaltenbacher, I. Shevchenkoa, and B. Wohlmuth, "A multi-time stepping integration method for the ultrasonic heating problem," Journal of the Acoustical Society of America, 2010. (submitted).

24. B. Kaltenbacher, T. Lahmer, M. Mohr, and M. Kaltenbacher, "PDE based determination of piezoelectric material tensors," European Journal of Applied Mathematics, vol. 17, pp. 383-416, 2006.

25. T. Lahmer, M. Kaltenbacher, B. Kaltenbacher, and R. Lerch, "FEM-Based Determination of Real and Complex Elastic, Dielectric and Piezoelectric Moduli in Piezoceramic Materials," IEEE Transactions on Ultrasonics, Ferroelectrics, and Frequency Control, vol. 55, no. 2, pp. 465-475, 2008. 
26. S. J. Rupitsch and R. Lerch., "Inverse method to estimate material parameters for piezoceramic disc actuators," Applied Physics A, vol. 97, no. 4, pp. :735-740, 2009.

27. J. Hoffelner, H. Landes, M. Kaltenbacher, and R. Lerch, "Finite Element Simulation of Nonlinear Wave Propagation in Thermoviscous Fluids Including Dissipation," IEEE Transactions on UFFC, vol. 48, pp. 779-786, May 2001.

28. M. Kaltenbacher, H. Landes, and R. Lerch, "An Efficient Calculation Scheme for the Numerical Simulation of Coupled Magnetomechanical Systems," IEEE Transactions on Magnetics, vol. 33, pp. 1646-1649, March 1997.

29. J. C. Hardin and M. Y. Hussaini, eds., Computational Aeroacoustics, ch. Computational aeroacoustics for low Mach number flows, pp. 50-68. Springer-Verlag, 1992. New York.

30. J. C. Hardin and M. Y. Hussaini, eds., Computational Aeroacoustics, ch. Regarding numerical considerations for computational aeroacoustics, pp. 216-228. Springer-Verlag, 1992. New York.

31. J. B. Freund, S. K. Lele, and P. Moin, "Direct Numerical Simulation of a Mach 1.92 Turbulent Jet and its Sound Field," AIAA Journal, vol. 38, pp. 2023-2031, 2000.

32. A. Uzun, A. S. Lyrintzis, and G. A. Blaisdell, "Coupling of Integral Acoustics Methods with LES for Jet Noise Prediction," in Proceedings of AIAA Aerospace Sciences Meeting and Exhibit, no. 2004-0517, January 2004. Reno, NV. USA.

33. M. E. Goldstein, Aeroacoustics. McGraw-Hill, 1976.

34. M. J. Lighthill, "On sound generated aerodynamically: I. General theory," Proc. Royal Society London, vol. 211, pp. 564-587, 1952.

35. N. Curle, "The influence of solid boundaries upon aerodynamic sound," Proc. Roy. Soc. London, vol. A 231, pp. 505-514, 1955.

36. J. Ffowcs-Williams and D. Hawkings, "Sound radiation from turbulence and surfaces in arbitrary motion," Phil. Trans. Roy. Soc., pp. 321-342, 1969.

37. K. Brentner and F. Farassat, "An analytical comparison of the acoustic analogy and Kirchhoff formulation for moving surfaces," AIAA Journal, vol. 36, no. 8, pp. 1379-1386, 1998.

38. F. Farassat, "Acoustic radiation from rotating blades - The Kirchhoff method in aeroacoustics," Journal of Sound and Vibration, vol. 239, no. 4, pp. 785 - 800, 2001.

39. P. Di Francescantonio, "A new boundary integral formulation for the prediction of sound radiation," Journal of Sound and Vibration, vol. 202, no. 4, pp. 491-509, 1997.

40. A. S. Lyrintzis, "Surface integral methods in computational aeroacoustics - From the (CFD) near field to the (Acoustic) far-field," Int. Journal of Aeroacoustics, vol. 2, no. 2, pp. 95-128, 2003.

41. D. Lockard, "A comparison of Ffowcs Williams-Hawkings Solvers for Airframe Applications," in Proceedings of 8th AIAA/CEAS Aeroacoustics Conference, June 2002. Breckenridge, USA.

42. F. Farassat and M. K. Myers, "Extension of Kirchhoff's formula to radiation from moving surfaces," Journal of Sound and Vibration, vol. 123, pp. 451-560, 1988.

43. A. Oberai, F. Ronaldkin, and T. Hughes, "Computational procedures for determining structuralacoustic response due to hydrodynamic sources," Comp. Methods Appl. Mech. Engineering, vol. 190, pp. 345-361, 2000.

44. C. Bogey, C. Bailly, and D. Juvé, "Computation of flow noise using source terms in linearized Euler's equations," AIAA Journal, vol. 40, no. 2, pp. 235-243, 2002.

45. J. C. Hardin and D. S. Pope, "An acoustic/viscous splitting technique for computational aeroacoustics," Theoretical and Computational Fluid Dynamics, vol. 6, pp. 323-340, 1994.

46. E. Manoha, C. Herrero, P. Sagaut, and S. Redonnet, "Numerical Prediction of Airfoil Aerodynamic Noise," in Proceedings of 8th AIAA/CEAS Aeroacoustics Conference, June 2002. Breckenridge, USA.

47. C. K. W. Tam and J. C. Webb, "Dispersion-Relation-Preserving Finite Difference Schemes for Computational Acoustics," Journal of Computational Physics, vol. 107, no. 2, pp. 262-281, 1993. 
48. W. D. Roeck, Hybride methodologies for computational aeroacoustic analysis of confined, subsonic flows. Ph.D., Catholic University of leuven, 2007.

49. M. Farshchi, S. Hannani, and M. Ebrahimi, "Lineraized and non-linear acoustic/viscous splitting techniques for low mach number flows," Int. J. Numer. Meth. Fluids, vol. 42, pp. 1059-1072, 2003.

50. R. Ewert and W. Schröder, "Acoustic Perturbation Equations based on Flow decomposition via Source Filtering," Journal of Computational Physics, vol. 188, pp. 365-398, 2003.

51. J. Seo and Y. Moon, "Linearized perturbed compressible equations for low Mach number aeroacoustics," Journal of Computational Physics, vol. 218, no. 2, pp. 702-719, 2006.

52. A. Oberai, F. Ronaldkin, and T. Hughes, "Computation of Trailing-Edge Noise due to Turbulent Flow over an Airfoil," AIAA Aeroacoustics Journal, vol. 40, pp. 2206-2216, 2002.

53. M. Kaltenbacher, M. Escobar, I. Ali, and S. Becker, "Numerical Simulation of Flow-Induced Noise Using LES/SAS and Lighthill's Acoustics Analogy," International Journal for Numerical Methods in Fluids, 2009. (doi: 10.1002/fld.2123).

54. M. Breuer, Direkte Numerische Simulation und Large-Eddy Simulation turbulenter Strömungen auf Hochleistungsrechnern. Shaker Verlag, 2001.

55. M. E. Goldstein, "A Hybrid RANS/LES Approach for Predicting Jet Noise," in 12th AIAA/CEAS Aeroacoustics Conference, no. 2006-2631, 2006.

56. U. Paliath and P. J. Morris, "Prediction of Jet Noise From Circular Beveled Nozzles," in 11th AIAA Aeroacoustics Conference, no. 2005-3096, 2005. Monterey, USA.

57. F. Menter and Y. Egorov, "A Scale Adaptive Simulation Model using Two-Equation Models," in 43rd AIAA Aerospace Sciences Meeting and Exhibit, no. AIAA-2005-1095. Reno, Nevada, Jan. 10-13, 2005.

58. C. Bailly and D. Juve, "A Stochastic Approach to Compute Subsonic Noise Using Linearized Euler's Equations," in AIAA-Paper 99-1872, 1999.

59. C. Tam and L. Auriault, "Jet Mixing Noise from Fine-Scale Turbulence," AIAA, vol. 37, no. 4, pp. 145-153, 1999.

60. M. Billson, L. Eriksson, and L. Davidson, "Jet noise prediction using stochastic turbulence modeling," in AIAA-Paper 2003-3282, 2003.

61. R. Ewert, "Broadband slat noise prediction based on caa and stochastic sound sources from a fast random particle-mesh (rpm) method," Computers \& Fluids, vol. 37, no. 4, pp. 369-387, 2008.

62. M. Bauer and R. Ewert, "Slat Noise Prediction using Discontinuous Galerkin Method and Stochastic Turbulent Sound Source," in AIAA 2010-3832, 2010.

63. C. Wagner, T. Hüttl, and P. Sagaut, eds., Large-Eddy Simulation for Acoustics. Cambridge University Press, 2007.

64. H. S. Ribner, "Effect of jet flow on jet noise via an extension to the Lighthill model," Journal of Fluid Mechanics, vol. 321, pp. 1-24, 1996.

65. G. M. Lilley, "The Generation and Radiation of Supersonic Jet Noise. Theory of Turbulence Generated Jet Noise, Noise Radiation from Upstream Sources, and Combustion Noise. Part II: Generation of Sound in a Mixing Region," Tech. Rep. 4, U.S. Air Force Aeropropulsion Lab., 1972. Vol. 4, AFAPL-TR-72-53.

66. G. M. Lilley, "On the noise from jets," tech. rep., AGARD, 1974. CP-131.

67. C. Bernardi, Y. Maday, and F. Rapetti, "Basics and some applications of the mortar element method," GAMM-Mitt. 28, vol. 2, pp. 97-123, 2005.

68. B. I. Wohlmuth, "A mortar finite element method using dual spaces for the lagrange multiplier," SIAM Journal on Numerical Analysis, vol. 38, no. 3, pp. 989-1012, 2001.

69. M. Escobar, Finite Element Simulation of Flow-Induced Noise using Lighthills Acoustic Analogy. PhD thesis, Technische Fakultät, Universität Erlangen-Nürnberg, 2007. 
March 11, $2011 \quad$ 8:4 WSPC/130-JCA CoupledFieldsAcousticEnd

36 M. Kaltenbacher

70. T. Belytschko, W. Liu, and B. Moran, Nonlinear Finite Elements for Continua and Structures. Chichester: J. Wiley \& Sons Ltd, 2000.

71. J. Donea and A. Huerta, Finite element methods for flow problems. Wiley, 2003.

72. G. Link, A Finite Element Scheme for Fluid-Solid-Acoustics Interactions and its Application to Human Phonation. PhD thesis, Technisch Fakultät Universität Erlangen-Nürnberg, 2008.

73. G. Link, M. Kaltenbacher, M. Breuer, and M. Döllinger, "A 2d finite-element scheme for fluidsolid-acoustic interactions and its application to human phonation," Computer Methods in Applied Mechanics and Engineering, vol. 198, pp. 3321-3334, 2009. 